\title{
Article \\ On-Line Learning and Updating Unmanned Tracked Vehicle Dynamics
}

\author{
Natalia Strawa ${ }^{\dagger}$, Dmitry I. Ignatyev *(D), Argyrios C. Zolotas $(\mathbb{D}$ and Antonios Tsourdos (1) \\ Centre for Autonomous and Cyber-Physical Systems, SATM, Cranfield University, Cranfield MK43 0AL, UK; \\ strawa.natalia@gmail.com (N.S.); a.zolotas@cranfield.ac.uk (A.C.Z.); a.tsourdos@cranfield.ac.uk (A.T.) \\ * Correspondence: d.ignatyev@cranfield.ac.uk \\ + Current Address: Hyper Poland, 03-828 Mazowieckie, Poland.
}

Citation: Strawa, N.; Ignatyev, D.I.; Zolotas, A.C.; Tsourdos, A. On-Line Learning and Updating Unmanned Tracked Vehicle Dynamics. Electronics 2021, 10, 187. https://doi.org/ 10.3390 /electronics10020187

Received: 13 October 2020 Accepted: 11 January 2021 Published: 15 January 2021

Publisher's Note: MDPI stays neutral with regard to jurisdictional clai$\mathrm{ms}$ in published maps and institutional affiliations.

Copyright: (C) 2021 by the authors. Licensee MDPI, Basel, Switzerland. This article is an open access article distributed under the terms and conditions of the Creative Commons Attribution (CC BY) license (https:// creativecommons.org/licenses/by/ $4.0 /)$.

\begin{abstract}
Increasing levels of autonomy impose more pronounced performance requirements for unmanned ground vehicles (UGV). Presence of model uncertainties significantly reduces a ground vehicle performance when the vehicle is traversing an unknown terrain or the vehicle inertial parameters vary due to a mission schedule or external disturbances. A comprehensive mathematical model of a skid steering tracked vehicle is presented in this paper and used to design a control law. Analysis of the controller under model uncertainties in inertial parameters and in the vehicle-terrain interaction revealed undesirable behavior, such as controller divergence and offset from the desired trajectory. A compound identification scheme utilizing an exponential forgetting recursive least square, generalized Newton-Raphson (NR), and Unscented Kalman Filter methods is proposed to estimate the model parameters, such as the vehicle mass and inertia, as well as parameters of the vehicle-terrain interaction, such as slip, resistance coefficients, cohesion, and shear deformation modulus on-line. The proposed identification scheme facilitates adaptive capability for the control system, improves tracking performance and contributes to an adaptive path and trajectory planning framework, which is essential for future autonomous ground vehicle missions.
\end{abstract}

Keywords: unmanned tracked vehicle; inertial parameters; vehicle-terrain interaction; identification; recursive least square with exponential forgetting; generalized Newton-Raphson; Unscented Kalman Filter

\section{Introduction}

The growing interest in the development of autonomous platforms establishes new research directions that address challenges associated with autonomous systems operations. Control of unmanned ground vehicles is a complex task due to vehicle-ground interactions that can strongly influence dynamics of the system and, at the same time, are very difficult to be measured or estimated analytically. Most of the important parameters may vary because a vehicle can cross different types of terrains during motion, mass and inertia can vary due to mission plan, etc. All these factors tend to influence control performance negatively and, as a result, trajectory tracking capabilities.

Learning and updating vehicle dynamics on the fly can tackle the aforementioned issues and improve the control and trajectory tracking performance, thus providing a framework for adaptive path-planning and control [1-4].

Usage of tracked vehicles in military, rescue, agricultural and recreational missions where terrain conditions are difficult or unpredictable is common. This is because tracked vehicles perform better than wheeled vehicles due to the larger contact area of tracks which provides better flotation and better mobility over unprepared terrain [3,5]. Hence, tracked vehicles are preferred choice for autonomous off-road tasks.

Skid steering can be characterized by an absence of a separate steering system, like, for example, in the Ackermann steering system. Advantages provided by the skid-steered scheme are a simple mechanical structure, robustness and high maneuverability [6]. 
Skid-steering, although energy inefficient, is commonly used for mobile robots (both wheeled and tracked) with the requirement of good mobility.

Skid-steering is performed by controlling relative velocities of the drives on the left and right sides of the vehicle; hence, track slippage causes turning. In this case, the motion of the tracked vehicle is governed by the two longitudinal track forces and the lateral friction force. Due to the fact that the friction force depends on the linear and angular velocities, the force equilibrium equation perpendicular to the tracks becomes a non-integrable differential equality constraint [3]. Maneuvering capabilities also depend on the complex vehicle-ground interaction [7].

For identification of varying parameters in real-time applications, kinematic models of vehicles seems more attractive as they put a less computational burden on the on-board instrumentation at the expense of unmodeled dynamic effects. Unfortunately, such simplification might be especially inefficient in case of skid-steering due to significant slippage. Some authors addressed this problem in their researches. For example, Wu et al. [7] proposed a method for experimental estimation of the wheeled vehicle kinematics. In their work, they applied approximating function with identifiable parameters to derive the relationship between the instantaneous center of rotation of the vehicle, its speed and path curvature. A different approach was studied by Sutoh et al. [8], who experimentally obtained the relationship between a rover's wheels input and output velocities for various types of loose terrain such as silica or gravel.

The biggest challenge in the ground vehicle dynamics modeling is a description of the vehicle-ground interaction. For the wheeled vehicles, model obtained experimentally by Pacejka [9] and so-called 'Pacejka's magic formula' is most widely used. In the case of tracked vehicles, the majority of authors applies the work of Wong [5]. A workaround for contact modeling problem was demonstrated in Reference [10], where a tyre-model-free integral control method correcting the wheel slip coefficient in real-time was designed. Many researches, however, utilize the classic dynamics model, including contact forces obtained with analytical functions derived from experimental data. Equations of motion for skid-steering based mobile robots are well-known and can be found to name a few in Reference [6,11-13]. Ahmadi et al. [14] slightly simplified the model by excluding the nonholonomic constraint from the dynamics equations, while Tang et al. [15] considered more complex and generalized track-terrain interactions model. Some advanced methods were also used to describe vehicle-terrain interaction. For example, Economou and Colyer [16] utilized the Fuzzy Logic for modeling of the vehicle-ground interactions based on the experimental results obtained for an electric wheeled skid steer vehicle under steady-state conditions and a variety of motions and surfaces. A neural network was employed to model the steering dynamics of an autonomous vehicle in Reference [17].

The path tracking control problem of a skid-steered vehicle is a fairly well-researched field. Hence, a variety of approaches can be found in the existing literature. Due to the highly nonlinear nature of the considered class of ground vehicles, a common starting point for the trajectory tracking control design is the feedback linearization. Thus, a linear control law can be further applied. The feedback linearization is thoroughly described in Reference [18]. A similar approach was used in Reference [11], and an exponentially stabilizing state feedback was further applied to complete the control design. Feed-forward friction compensation is implemented alongside the feedback linearization in Reference [14]. Their controller is based on the simplified model of the tracked vehicle. The elaborated forceslip relationship is linearized to relate the inputs with the states of the system. A robust recursive LQR design was applied for the mobile robot in Reference [19]. A neural network model of the steering dynamics of an autonomous vehicle developed in Reference [17] was integrated with a Nonlinear Model Predictive Controller to generate feed-forward steering commands. Recently, approaches based on dynamic model of vehicle for optimal path planning and tracking control of unmanned ground vehicles (UGV) were proposed in Reference [20,21]. Moreover, a novel approach introduced in Reference [22] employs a backstepping technique to robustly control the instantaneous center of rotation position 
of the vehicle so that it relates to the path curvature and the desired vehicle speed. The backstepping method was also utilized by Zou et al. [13], who designed a modified PID computed-torque controller for an unmanned tracked vehicle.

From a practical point of view, the interest of the current research lays in identification of the following parameters: mass, inertia, and slip, as well as the soil parameters, as part of the vehicle-terrain interaction model.

Vehicle mass and inertia estimations become increasingly important with the rising popularity of autonomous vehicles. These parameters are especially vital in the heavy-duty vehicles automation, powertrain and economic cruise control [23,24]. Upon the analysis of approaches presented in the existing literature, exponential forgetting recursive least squares (EF RLS) method is the most widely used for this purpose [23-25] since it is a very powerful and robust method when the system dynamics can be represented in a linear form. A different method for vehicle mass estimation was introduced by Rhode and Gauterin [26], who utilized the total least squares (TLS) regression.

Regarding the slip estimation, although it is possible to measure all the quantities essential for obtaining the slip value from analytical expressions, measurement inaccuracies and errors lead to the results highly inconsistent with the real slip values. As the slip estimation problem has a significant impact on the tracked vehicle control, therefore, many researchers sought the solution.

Dar and Longoria [27] utilized an Extended Kalman Filter (EKF) with state noise compensation to estimate slip, trajectory and orientation of a small tracked vehicle. However, some authors question the accuracy of EKF estimation, especially in the case of the fast dynamics. Instead, they suggest employing an Unscented Kalman Filter (UKF) which can provide more accuracy without linearization [28]. In research [29], not only kinematics of the vehicle but also simplified dynamics were included in the UKF design. Alternatively, a sliding mode observer (SMO) was exploited in Reference [30] to obtain an accurate slip estimation. In their comparative studies, the authors proved that both the UKF and SMO yield better results than those obtained with the EKF.

Another important vehicle dynamics parameter is the maximum tractive effort that certain vehicle can develop on certain soil types. This property has a major influence on path planning as it can restrict the maneuverability of the vehicle and has a huge impact on the energy efficiency [5]. For satisfactory estimation of soil parameters the NewtonRaphson (NR) method is widely used [31-33]. In particular, in Reference [31], the authors conducted a comparison between NR and RLS that manifested that the former yields much better estimation accuracy and robustness as RLS tends to diverge quite often as it is more prone to the measurement noise.

UGVs are characterized by nonlinear dynamics and excited by a combination of multiple external and internal (to the system) factors. Even small variation (and/or uncertainties) in parameters of a nonlinear system can cause dramatic departures from a "nominal" case scenario. Although many research works focused on designing algorithms for the identification of a particular parameter of UGV dynamics, there is a lack of studies addressing the whole (integrated) dynamics or, at least, its major part. A rigorous evaluation of the system performance is required, especially, while traversing unknown terrains (as is the case investigated in this paper). Such an integrated estimator-based approach is extremely valuable for the development of advanced autonomous systems utilizing AI tools to estimate and predict vehicle behavior for uncertain in nature terrain environments and generate optimal path $[2,34,35]$.

Thus, the main aim of this research was the development of an integrated system running on-line algorithms estimating different vehicle model parameters. To reach this aim, the simplicity and computational efficiency were prioritized. Influence of uncertainties of the dynamics model used for the control design on the behavior of an autonomous tracked vehicle was studied. On-line system identification algorithms using the EF RLS, UKF, and NR algorithms were implemented for soil and inertial parameters estimation; 
their performance was further evaluated. The system considered in this study consists of the tracked vehicle model, trajectory tracking controller, and the identification module.

This paper is organized as follows. Section 2 provides description of the track-terrain interaction model. The kinematic and dynamic models of the tracked vehicle are given in Sections 3 and 4, correspondingly. Overview of the entire system is provided in Section 5. Design of the control system is described in Section 6. The proposed identification framework is demonstrated in Section 7. The obtained results are discussed in Section 8, and, finally, Section 9 concludes the paper.

\section{Track-Terrain Interaction Model}

The system identification has the following key elements, namely selection of the model structure, experiment design, and parameter estimation. On-line identification is performed automatically, so it is extremely important to have a good understanding of all aspects of the problem [1]. That is why we provide here a detailed description of vehicle dynamics, including vehicle-terrain interaction model.

\subsection{Terramechanics}

The current section gives a brief introduction into terramechanics to equip with a basic knowledge of the track-terrain interaction, indispensable for the tracked vehicle modeling.

Mobility of the off-road vehicles can be severely limited by properties of the encounter terrain. Terramechanics provides essential knowledge of the mechanical properties of the terrain, as well as its reaction to vehicular loadings.

Certain types of trafficable terrain can be considered as ideal elastoplastic materials [5]. Unless stress level exceeds a given limit, terrain behavior remains in the elastic range (see Figure 1). This assumption is utilized for stress distribution prediction in the soil. When yield stress is reached in the terrain under vehicular load, strain increases and plastic flow is constituted. Transition to plastic flow is known as the failure of soils. Failure criterion can be described with the Mohr-Coulomb theory, which postulates that the following condition must be satisfied at the point of the material to cause its failure [36]:

$$
\tau=c+\sigma \tan \phi,
$$

where $\tau$ is the shear stress, $c$ is cohesion, $\sigma$ is normal stress on the sheared surface, and $\phi$ is the angle of internal shearing resistance.

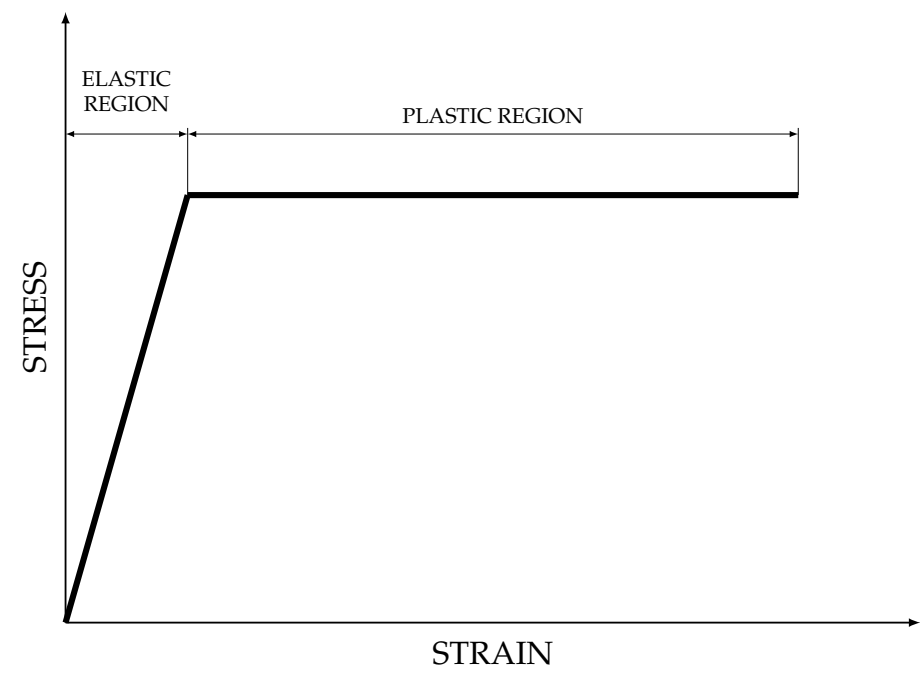

Figure 1. Strain-stress relation of idealized elastoplastic material, adopted from Reference [36].

Cohesive forces bind soil grains together irrespective of normal pressure between the particles, e.g., for saturated clay only cohesion $c$ is presented. However, when grains are not held together by cohesion, they can move upon each other and, while pressed against 
each other, friction develops, e.g., for dry sand, the shear strength can be expressed by $\sigma \tan \phi$. In practice, the majority of the soils exhibit both plastic and frictional behavior and the shear strength should be hence characterized with Equation (1).

\subsection{Tractive Effort and Slip of a Track}

For a mathematical description of a ground vehicle motion, a track-terrain interaction should be considered. In the current study, a parametric approach proposed by Bekker [36] is used to do this.

Bekker's method assumes that a track in contact with a terrain is similar to a rigid footing. Moreover, it is assumed in the current research, that the center of gravity (CG) of the tracked vehicle is positioned at the mid-point of the track-terrain contact area. It is also assumed here that a normal pressure produced by the track has an uniform distribution along the track.

During the vehicle motion a torque produced by the motors and applied to the sprocket of the track initiates shearing action on the track-terrain interface, which subsequently results in a development of tractive effort. The maximum tractive effort is bounded by the maximum shear strength of the soil $\tau_{\max }$ and the track contact area $A$. Taking into account Equation (1), we can obtain:

$$
\begin{aligned}
F_{\max } & =A \tau_{\max } \\
& =A(c+p \tan \phi) \\
& =A c+W \tan \phi,
\end{aligned}
$$

where $A=b l, b$ is the contact width, $l$ is the contact length, $W$ is the normal load, and $c$ and $\phi$ are the soil parameters: the cohesion and the angle of internal shearing resistance, respectively. It can be observed that the terrain type critically impacts the maximum shear strength of the soil and, consequently, has great influence on the maximum tractive effort. For instance, as it was mentioned before, dry sand is a frictional soil. Therefore, cohesion is negligible in this case and maximum tractive effort is higher for heavier vehicles. On the other hand, the saturated clay, which is an example of cohesive soil, has a low value of $\phi$; hence, mainly the contact area of the track influences the maximum tractive effort value.

It should be pointed out that the tractive effort defined in Equation (2) is a maximum value that the tracked vehicle can develop on a certain terrain. To determine thrust over a full operating range, its relationship with the slip of a track should be examined. The slip of a track is defined as follows [5]:

$$
i=1-\frac{V}{\omega r}=1-\frac{V}{V_{t}}=\frac{V_{t}-V}{V_{t}}=\frac{V_{j}}{V_{t}}
$$

where $V$ is the forward speed of a track, and $V_{t}$ is the theoretical speed defined by the sprocket rotational speed $\omega$ and its radius $r$. Then, $V_{j}$ is the speed of slip with reference to the ground. Assuming that the track is rigid and cannot stretch, the vehicle is moving along a flat surface with a homogeneous soil property, then every point of the track, which is in contact with the terrain, has the same speed $V_{j}$. Therefore, the shear displacement $j$ at a distance $x$ from the front of the track-terrain contact area can be found with the following equation:

$$
j=V_{j} t
$$

with $t=x / V_{t}$ being the contact time of a considered point and the terrain. Thus, Equation (4) can be rearranged:

$$
j=\frac{V_{j} x}{V_{t}}=i x .
$$

Equation (5) indicates that the shear displacement increases linearly with the distance from the front of the contact area (see Figure 2). 


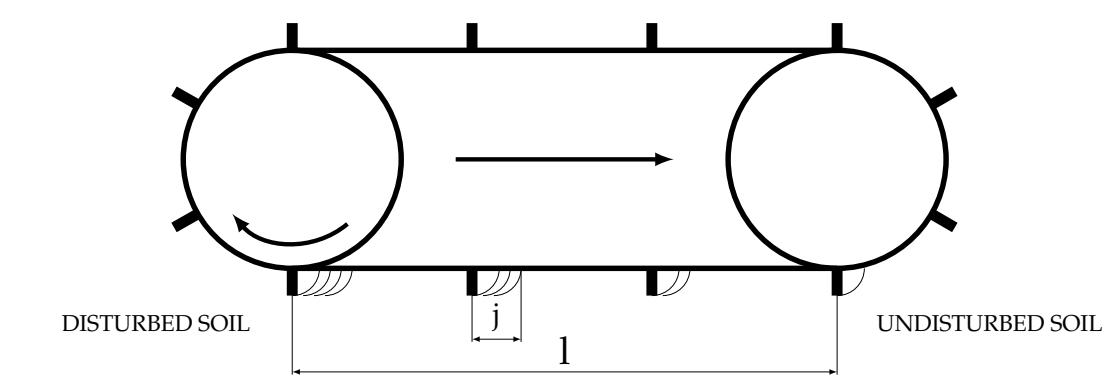

Figure 2. Shear displacement developing under a track [5].

For plastic soils, which have shear stress-displacement relationship described by the simple exponential equation proposed by by Janosi and Hanamoto [37], development of the shear stress is directly related to shear displacement and can be defined with the following function:

$$
\tau=\tau_{\max }\left(1-e^{-j / K}\right)=(c+\sigma \tan \phi)\left(1-e^{-j / K}\right),
$$

where $K$ is the soil shear deformation modulus. Since we assumed the uniform normal pressure distribution $(\sigma=W / b l)$, which is independent of $x$, the total tractive effort of the track can be represented in the following form:

$$
\begin{aligned}
F & =b \int_{0}^{l} \tau d x \\
& =b \int_{0}^{l}(c+\sigma \tan \phi)\left(1-e^{-j / K}\right) d x \\
& =b \int_{0}^{l}\left(c+\frac{W}{b l} \tan \phi\right)\left(1-e^{-j / K}\right) d x \\
& =(A c+W \tan \phi)\left[1-\frac{K}{i l}\left(1-e^{-i l / K}\right)\right] .
\end{aligned}
$$

The advantage of the proposed friction model is that it provides high predictive capabilities while maintaining low computational complexity. This is a quite strong point while designing the considered framework, which is focused on the integration of different on-line identification algorithms running at the same time on-board.

\section{Kinematics}

Kinematics equations relates rotation of the track sprockets with the vehicle motion. Here, we are considering kinematics of a maneuvering planar skid-steering vehicle. Two orthonormal bases are introduced: the inertial frame $(X, Y, Z)$ and the body-fixed frame $(x, y, z)$ with its origin at the center of mass (COM) of the vehicle (Figure 3$)$. Note that $Z$ coordinate remains constant as the vehicle is in planar motion. Moreover, to simplify the formulation, it is assumed that COM coincides with the centroid of the vehicle body.

Let us assume that the vehicle moves with a linear velocity:

$$
\mathbf{v}_{\mathcal{B}}=\left[\begin{array}{lll}
\dot{x} & \dot{y} & 0
\end{array}\right]^{T},
$$

expressed in the local frame $\mathcal{B}$ and rotates with the angular velocity:

$$
\boldsymbol{\omega}=\left[\begin{array}{lll}
0 & 0 & \dot{\theta}
\end{array}\right]^{T} .
$$

We choose the generalized coordinate vector as follows:

$$
\mathbf{q}=\left[\begin{array}{lll}
X & Y & \theta
\end{array}\right]^{T}
$$


where $\theta$ is the orientation of the local coordinate frame $\mathcal{B}$ with respect to the inertial frame $\mathcal{I}$. As a result, the vector of generalized velocities is defined as:

$$
\dot{\mathbf{q}}=\left[\begin{array}{lll}
\dot{X} & \dot{Y} & \dot{\theta}
\end{array}\right]^{T} .
$$

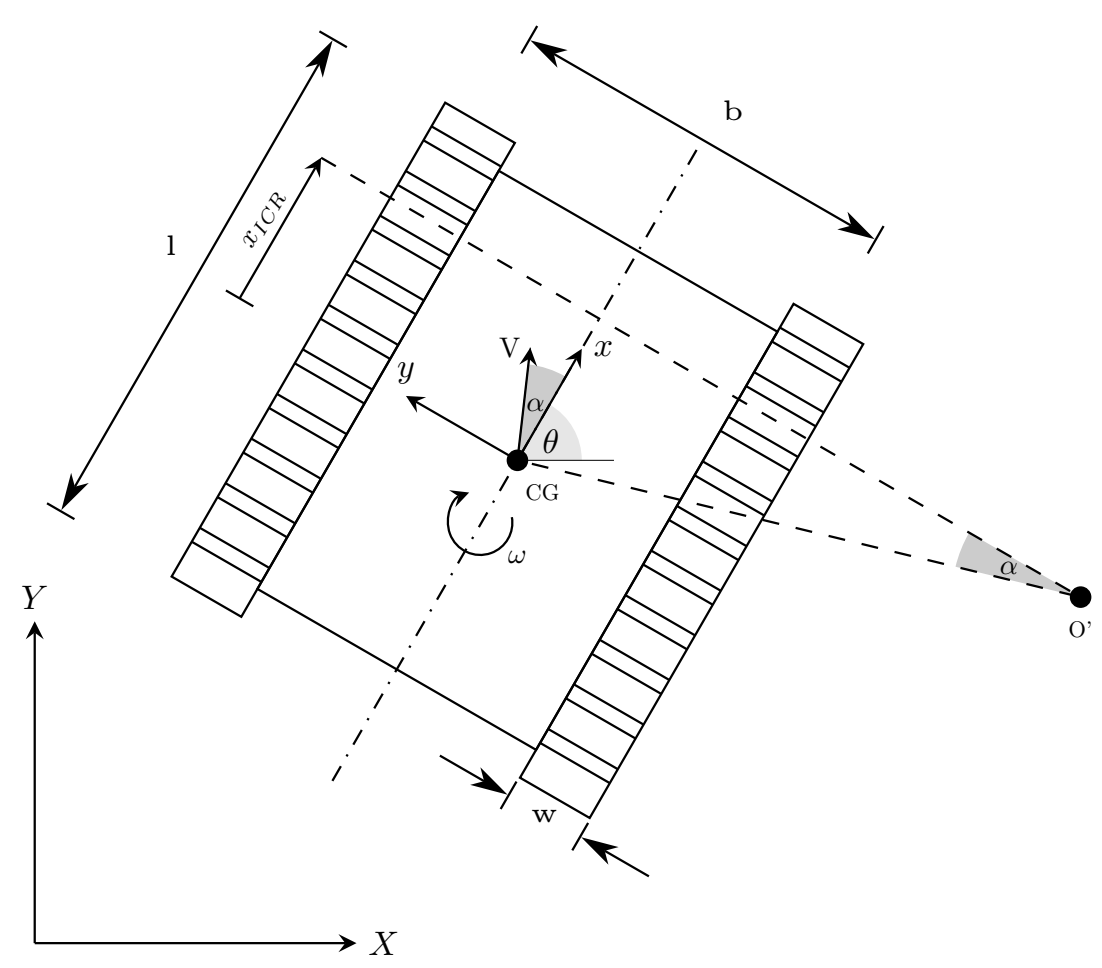

Figure 3. Kinematics of a tracked vehicle.

Here, we introduce rotation matrix $\mathbf{R}_{2 \times 2}$ that carries $\mathcal{I}$ into $\mathcal{B}$ [13]:

$$
\mathbf{R}(\theta)=\left[\begin{array}{cc}
\cos \theta & -\sin \theta \\
\sin \theta & \cos \theta
\end{array}\right]
$$

In what follows, $\sin (\cdot)$ and $\cos (\cdot)$ are denoted by $s(\cdot)$ and $c(\cdot)$, respectively, for simplicity of notation.

Thus, velocity of the vehicle can be expressed in the inertial frame by means of $\mathbf{v}_{B}$ and $\mathbf{R}(\theta)$ [11]:

$$
\left[\begin{array}{c}
\dot{X} \\
\dot{Y}
\end{array}\right]=\mathbf{R}(\theta) \mathbf{v}_{B}=\left[\begin{array}{cc}
c \theta & -s \theta \\
s \theta & c \theta
\end{array}\right]\left[\begin{array}{c}
\dot{x} \\
\dot{y}
\end{array}\right] .
$$

We assume that, when maneuvering, slipping or skidding are possible. When these effects are taken into consideration, the vehicle speed can be obtained from the following expression [13]:

$$
V=\frac{r}{2 \cos \alpha}\left[\omega_{L}\left(1-i_{L}\right)+\omega_{R}\left(1-i_{R}\right)\right]
$$

where $\alpha$ is a slip angle, $r$ is the radius of the sprockets, and $\omega_{L}, \omega_{R}$, and $i_{L}, i_{R}$ denote the left and the right track rotational velocities and slips, respectively. The rotational velocity can be calculated using the following equation:

$$
\dot{\theta}=\frac{r}{b}\left[\omega_{L}\left(1-i_{L}\right)-\omega_{R}\left(1-i_{R}\right)\right] .
$$

Slip of the left and right track is given by Reference [5]: 


$$
\begin{aligned}
& i_{L}=1-\frac{v_{t, L}}{\omega_{L} r}=1-\frac{\dot{x}+(b / 2) \dot{\theta}}{\omega_{L} r}, \\
& i_{R}=1-\frac{v_{t, R}}{\omega_{L} r}=1-\frac{\dot{x}-(b / 2) \dot{\theta}}{\omega_{R} r},
\end{aligned}
$$

and the slip angle can be computed as follows:

$$
\alpha=\arctan \frac{\dot{y}}{\dot{x}} .
$$

Taking into account Equations (8) and (9), the turning radius $R$, with slip considered, can be obtained:

$$
R=\frac{V}{\dot{\theta}}=\frac{b}{2 \cos \alpha} \frac{\omega_{L}\left(1-i_{L}\right)+\omega_{R}\left(1-i_{R}\right)}{\omega_{L}\left(1-i_{L}\right)-\omega_{R}\left(1-i_{R}\right)} .
$$

Since velocity in the body frame can be expressed through speed and slip angle the following equation can be obtained:

$$
\left[\begin{array}{c}
\dot{x} \\
\dot{y}
\end{array}\right]=V\left[\begin{array}{l}
\cos \alpha \\
\sin \alpha
\end{array}\right] .
$$

Therefore, this approach can be further projected to the inertial frame

$$
\left[\begin{array}{l}
\dot{X} \\
\dot{Y}
\end{array}\right]=V\left[\begin{array}{l}
c \theta c \alpha-s \theta s \alpha \\
s \theta c \alpha+c \theta s \alpha
\end{array}\right]
$$

and, subsequently, one can relate the velocity in the $\mathcal{I}$ frame with the rotational velocities of the sprockets and slips of the tracks, which yields a complete kinematic model of the tracked vehicle [13]:

$$
\begin{aligned}
& \dot{X}=\frac{r}{2}\left[\omega_{L}\left(1-i_{L}\right)+\omega_{R}\left(1-i_{R}\right)\right][c \theta-s \theta \tan \alpha], \\
& \dot{Y}=\frac{r}{2}\left[\omega_{L}\left(1-i_{L}\right)+\omega_{R}\left(1-i_{R}\right)\right][s \theta+c \theta \tan \alpha], \\
& \dot{\theta}=\frac{r}{b}\left[\omega_{L}\left(1-i_{L}\right)-\omega_{R}\left(1-i_{R}\right)\right] .
\end{aligned}
$$

To finalize the mathematical description of the kinematics model of the tracked vehicle, nonholonomic constraint should be imposed [12,14]. The arbitrary planar motion of a body can be represented as a rotation around the instantaneous center of rotation (ICR) [38]. This concept applied to the tracked vehicle is demonstrated in Figure 4. ICR of the tracked vehicle is denoted by $O^{\prime}$, while the rotation radius vectors, defined in the body-fixed frame and directed from the ICR, are: $\mathbf{d}_{t, i}=\left[\begin{array}{ll}d_{t, i x} & d_{t, i y}\end{array}\right]^{T}$, where $i=\{L, R\}$, and $\mathbf{d}_{C}=\left[\begin{array}{ll}d_{C x} & d_{C y}\end{array}\right]^{T}$. From the definition of ICR, we can obtain

$$
\frac{\left\|\mathbf{v}_{t, i}\right\|}{\left\|\mathbf{d}_{t, i}\right\|}=\frac{\|\mathbf{V}\|}{\left\|\mathbf{d}_{C}\right\|}=|\omega|,
$$

where $\|\cdot\|$ is the Euclidean norm. Equation (13) can be further transformed to the expanded form

$$
\frac{v_{t, i x}}{-d_{t, i y}}=\frac{V_{x}}{-d_{C y}}=\frac{v_{t, i y}}{d_{t, i x}}=\frac{V_{y}}{d_{C x}}=\omega .
$$




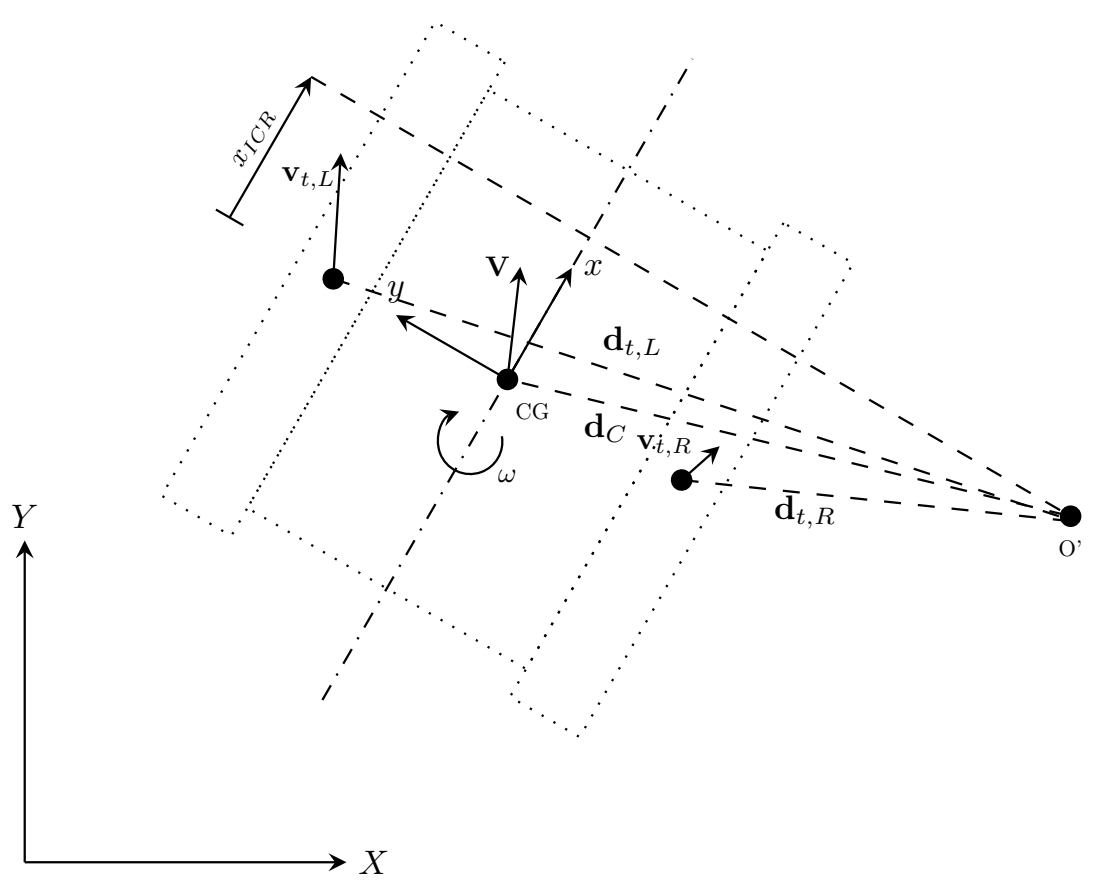

Figure 4. Instantaneous center of rotation geometry.

Coordinates of the ICR can be defined in the local frame as

$$
I C R=\left(x_{I C R}, y_{I C R}\right)=\left(-d_{C x},-d_{C y}\right) .
$$

Thus, Equation (14) can be rewritten in the following way:

$$
\frac{V_{x}}{y_{I C R}}=-\frac{V_{y}}{x_{I C R}}=\omega .
$$

Note that, due to the planar motion of the vehicle, $\omega=\dot{\theta}$.

The ICR coordinates can be obtained from Equation (16) as follows:

$$
\left[\begin{array}{c}
x_{I C R} \\
y_{I C R}
\end{array}\right]=\left[\begin{array}{c}
-\dot{y} / \dot{\theta} \\
\dot{x} / \dot{\theta}
\end{array}\right]
$$

In a case of straight line motion, both the lateral velocity $\dot{y}$ and the angular velocity $\dot{\theta}$ vanish; thus, the ICR shifts to infinity along the y-axis. During turning maneuvers, the ICR moves along $\mathrm{x}$-axis by an amount of $\left|x_{I C R}\right|$. Shift of the $x_{I C R}$ beyond the vehicle geometry causes loss of motion stability.

Following Reference [11], we impose the nonholonomic constraint obtained from Equation (16)

$$
V_{y}+x_{I C R} \dot{\theta}=0 .
$$

The above expression can be presented in Pfaffian form [39]

$$
\left[\begin{array}{lll}
-\sin \theta & \cos \theta & x_{I C R}
\end{array}\right]\left[\begin{array}{c}
\dot{X} \\
\dot{Y} \\
\dot{\theta}
\end{array}\right]=\mathbf{A}(\mathbf{q}) \dot{\mathbf{q}}=\mathbf{0} .
$$

Now, we choose a full-rank matrix $\mathbf{S}(\mathbf{q}) \in \mathbb{R}^{3 x 2}$, in which columns are in null space of $\mathbf{A}(\mathbf{q})$, that is

$$
\mathbf{S}^{T}(\mathbf{q}) \mathbf{A}(\mathbf{q})=\mathbf{0},
$$


and $\mathbf{S}(\mathbf{q})$ can be, for example, defined as follows:

$$
\mathbf{S}(\mathbf{q})=\left[\begin{array}{cc}
c \theta & x_{I C R} s \theta \\
s \theta & -x_{I C R} c \theta \\
0 & 1
\end{array}\right] .
$$

Then, it is possible to define the generalized velocities by means of $\mathbf{S}$ and an auxiliary vector $\boldsymbol{v}(t) \in \mathbb{R}^{2}$

$$
\dot{\mathbf{q}}=\mathbf{S}(\mathbf{q}) \boldsymbol{v}(t),
$$

where the auxiliary vector is $\boldsymbol{v}(t)=\left[\begin{array}{ll}V & \dot{\theta}\end{array}\right]^{T}$.

\section{Dynamics}

In this section, we provide a model of tracked vehicle dynamics for a comprehensive description of the vehicle dynamics required for the development of the controller and identification scheme.

\subsection{Forces and Moments Acting on the Tracked Vehicle}

In the current analysis, we assume that the service brake is not applied, and the friction brakeforce is not generated. Additionally, the aerodynamic loads are neglected since the vehicle travels with a very low speed and the cross-sectional area of the vehicle is assumed to be small.

A moving vehicle is subjected only to track-terrain interaction forces, which are illustrated in Figure 5 and can be classified as follows:

- tractive forces $\mathbf{F}_{R}$ and $\mathbf{F}_{L}$,

- longitudinal resistance forces $\mathbf{R}_{R}$ and $\mathbf{R}_{L}$,

- lateral forces $\mathbf{F}_{y, R}$ and $\mathbf{F}_{y, L}$, and

- moment of turning resistance $\mathbf{M}_{r}$ induced by the resistive forces.

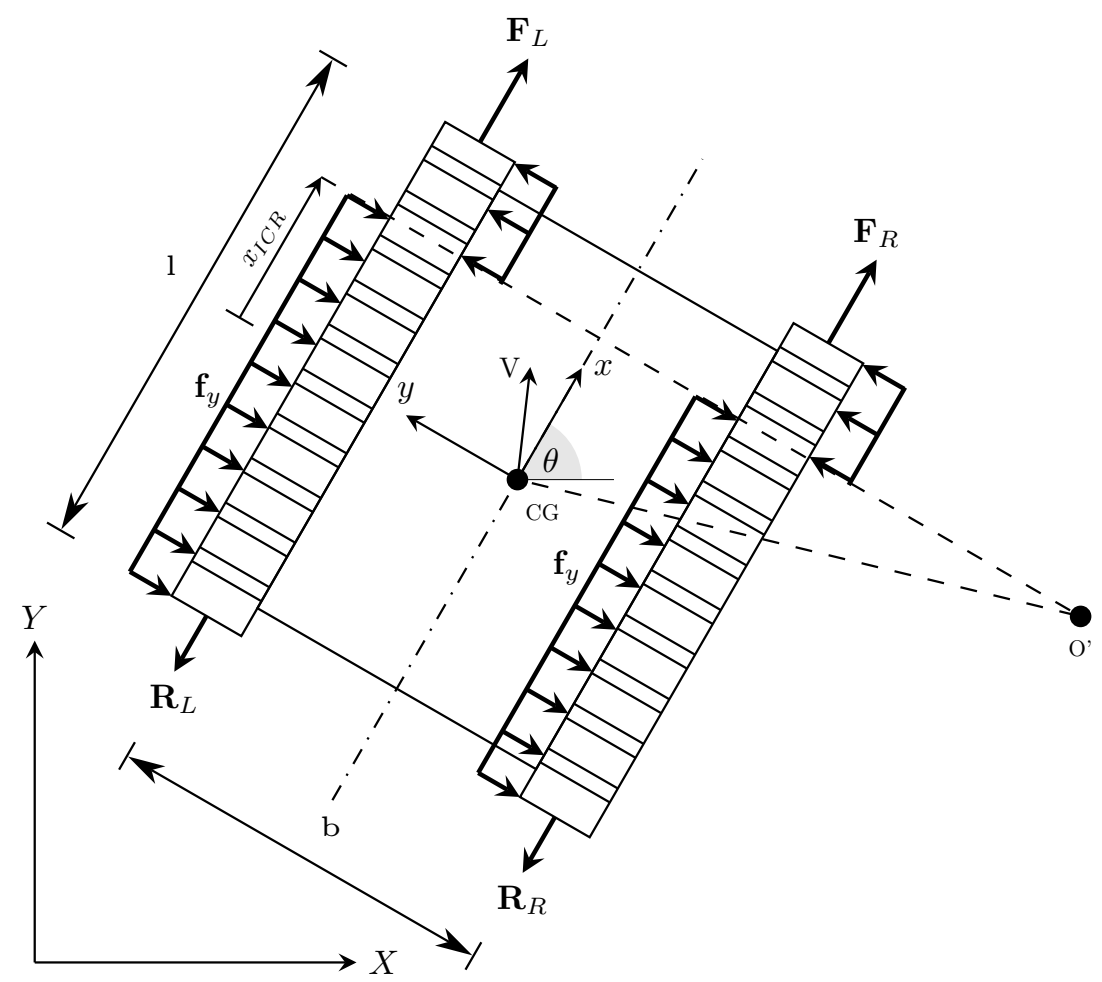

Figure 5. Free-body diagram. 


\subsubsection{Tractive Force}

The concept of tractive force has been already discussed in Section 2.1. In this study, it is assumed that rotation of the tracks, which results in the development of traction, is caused by the torque transmitted to the sprockets from a pair of DC motors-one per each track of the vehicle. Transmission factor is assumed to be ideal.

\subsubsection{Longitudinal and Lateral Resistance Forces}

Friction term for longitudinal resistance force can be calculated with the following expression [5]:

$$
r_{l}=\frac{\mu_{l} m g}{2},
$$

where $\mu_{l}$ is the coefficient of longitudinal resistance, and $g$ is the gravitational acceleration. To define the direction of the longitudinal friction forces, the following function is employed [14]:

$$
G(F, f, \dot{x})= \begin{cases}-f \operatorname{sign}(\dot{x}), & \dot{x} \neq 0, \\ 0, & \dot{x}=0,|F| \leq f, \\ -f \operatorname{sign}(F), & \dot{x}=0,|F|>f,\end{cases}
$$

where $\operatorname{sign}(\cdot)$ denotes the signum function.

Then, the longitudinal resistance force can be defined as follows:

$$
R_{l, i}=G\left(F_{i}, r_{l}, v_{t, i}\right), \quad i=\{R, L\} .
$$

The lateral friction distribution and force can be obtained according the following equations:

$$
f_{y}=\frac{\mu_{t} m g}{l}, \quad F_{y}=-2 \operatorname{sign}(\dot{y}) f_{y} x_{I C R},
$$

where $\mu_{t}$ denotes the coefficient of lateral resistance.

\subsubsection{Turning Moment and Moment of Turning Resistance}

The turning moment is induced by the forces acting in the longitudinal direction, i.e.,

$$
M=\left(F_{L}-R_{l, L}\right) \frac{b}{2}-\left(F_{R}-R_{l, R}\right) \frac{b}{2}=\left(F_{L}-F_{R}\right) \frac{b}{2} .
$$

The resistance moment can be obtained by integrating over the track length of the distribution given by the following expression from Reference [3]:

$$
m_{r}=2 f_{y}\left(\frac{l^{2}}{4}-x_{I C R}^{2}\right) .
$$

Direction of the moment of turning resistance can be again determined with Equation (19), namely

$$
M_{r}=G\left(M, m_{r}, \dot{\theta}\right) .
$$

\subsubsection{Drive Model}

In this study, it is assumed that the vehicle is actuated with two DC motors that drive the sprockets through the transmission gear and are controlled with a simple PID controller. The relationship between the motor torque $\tau$ and the rotor current $i_{a}$ is considered to be linear [12]:

$$
\tau_{m}=k_{m} i_{a}
$$

where $k_{m}$ is a motor torque constant. Voltage and current in the motor circuits can be approximated with the differential Equation [12]:

$$
u_{a}=L_{a} \frac{d}{d t} i_{a}+R_{a} i_{a}+k_{e} \omega_{m}
$$


where $L_{a}$ and $R_{a}$ are the inductance and resistance of the motor, respectively, $k_{e}$ denotes the electromotive force coefficient, and $\omega_{m}$ is the angular velocity at the output. Under the assumption that the transmission is ideal, we can write:

$$
\begin{aligned}
& \tau=n k_{m} i_{a}, \\
& \omega_{m}=n \omega_{s},
\end{aligned}
$$

where $n$ is the transmission ratio, and $\omega_{s}$ is the rotational speed of sprocket.

Assuming that the voltage $u_{a}$ is the motor control input, the following equations can describe the powertrain system:

$$
\begin{aligned}
\boldsymbol{\tau} & =n k_{m} \mathbf{i}_{a}, \\
\mathbf{u}_{a} & =L_{a} \frac{d}{d t} \mathbf{i}_{a}+R_{a} \mathbf{i}+k_{e} \boldsymbol{\omega}_{m},
\end{aligned}
$$

where $\boldsymbol{\tau}=\left[\begin{array}{ll}\tau_{L} & \tau_{R}\end{array}\right]^{T}, \mathbf{u}_{a}=\left[\begin{array}{ll}u_{L} & u_{R}\end{array}\right]^{T}, \mathbf{i}_{a}=\left[\begin{array}{ll}i_{L} & i_{R}\end{array}\right]^{T}$, and $\boldsymbol{\omega}=\left[\begin{array}{ll}\omega_{L} & \omega_{R}\end{array}\right]^{T}$.

\subsection{Equations of Motion}

Taking into account the nonholonomic constraint, we can obtain the equations of motion of the tracked vehicle through Lagrange-Euler formula with Lagrange's multipliers similar to approach given in Reference [40]:

$$
\frac{d}{d t}\left(\frac{\partial L}{\partial \dot{\mathbf{q}}}\right)-\frac{\partial L}{\partial \mathbf{q}}+\mathbf{A}(\mathbf{q}) \boldsymbol{\lambda}=\mathbf{Q}
$$

where $\lambda$ is vector of the Lagrange's multipliers, $\mathbf{A}(\mathbf{q})$ is the nonholonomic constraint defined in Equation (17), $\mathbf{Q}$ is the vector of generalized forces, and $L(\dot{\mathbf{q}}, \mathbf{q}, t)$ is the Lagrangian defined as

$$
L=E_{T}-E_{V}
$$

where $E_{T}$ and $E_{V}$ are the kinetic and potential energy, respectively.

First, we obtain the Lagrangian of the system. Since it is assumed that the vehicle is in planar motion, it can be assumed that $E_{V}=0$ and Equation (23) takes the following form:

$$
L(\dot{\mathbf{q}}, \mathbf{q}, t)=E_{T}(\dot{\mathbf{q}}, \mathbf{q}) .
$$

Assuming that the energy of rotating tracks can be neglected, the kinetic energy of the system is given by

$$
E_{T}=\frac{1}{2} m \mathbf{v}^{T} \mathbf{v}+\frac{1}{2} I \omega^{2},
$$

where $m$ is mass of the vehicle, and $I$ denotes its moment of inertia about the COM. As $\mathbf{v}^{T} \mathbf{v}=\|V\|^{2}$, and the value of velocity magnitude is independent of the reference frame, one can rewrite Equation (24) in the following form

$$
E_{T}=\frac{1}{2} m\left(\dot{X}^{2}+\dot{Y}^{2}\right)+\frac{1}{2} I \dot{\theta}^{2} .
$$

Hence, the derivatives of kinetic energy can be computed as

$$
\begin{gathered}
\frac{d}{d t}\left(\frac{\partial E_{T}}{\partial \dot{\mathbf{q}}}\right)=\left[\begin{array}{c}
m \ddot{X} \\
m \ddot{Y} \\
I \ddot{\theta}
\end{array}\right]=\mathbf{M} \ddot{\mathbf{q}}, \\
\frac{\partial E_{T}}{\partial \mathbf{q}}=\mathbf{0},
\end{gathered}
$$


where

$$
\mathbf{M}=\left[\begin{array}{ccc}
m & 0 & 0 \\
0 & m & 0 \\
0 & 0 & I
\end{array}\right]
$$

Vector of the generalized forces can be decomposed into actuating forces generated by motors and resistive forces causing dissipation of energy, which yields

$$
\mathbf{Q}=\mathbf{B}(\mathbf{q}) \boldsymbol{\tau}-\mathbf{C}(\mathbf{q}, \dot{\mathbf{q}}),
$$

with

$$
\mathbf{C}(\mathbf{q}, \dot{\mathbf{q}})=\left[\begin{array}{c}
2 R_{l} c \theta+F_{y} s \theta \\
2 R_{l} s \theta-F_{y} c \theta \\
M_{r}
\end{array}\right], \quad \mathbf{B}(\mathbf{q})=\frac{1}{r}\left[\begin{array}{cc}
c \theta & c \theta \\
s \theta & s \theta \\
b / 2 & -b / 2
\end{array}\right], \quad \boldsymbol{\tau}=\left[\begin{array}{c}
\tau_{L} \\
\tau_{R}
\end{array}\right],
$$

where $r$ is the sprocket radius, and $\tau_{L}, \tau_{R}$ are the torques provided by the left and right motors, respectively. Having all terms of Equation (22) defined, the mathematical model of system dynamics is obtained [11,13]:

$$
\mathbf{M} \ddot{\mathbf{q}}+\mathbf{C}(\mathbf{q}, \dot{\mathbf{q}})+\mathbf{A}(\mathbf{q}) \lambda=\mathbf{B}(\mathbf{q}) \tau
$$

For control purposes, it is convenient to express the generalized velocities $\dot{\mathbf{q}}$ in terms of pseudo-velocity $\boldsymbol{v}(t)$. Differentiating Equation (18), we have

$$
\ddot{\mathbf{q}}=\dot{\mathbf{S}}(\mathbf{q}) \boldsymbol{v}(t)+\mathbf{S}(\mathbf{q}) \dot{\boldsymbol{v}}(t)
$$

Next, Equations (18) and (27) are substituted into Equation (26), and both sides of the obtained equation are multiplied by $\mathbf{S}^{T}(\mathbf{q})$, which leads to the modified mathematical description of the system dynamics

$$
\begin{aligned}
& \dot{\mathbf{q}}=\mathbf{S}(\mathbf{q}) \boldsymbol{v}(t), \\
& \dot{\boldsymbol{v}}(t)=\widetilde{\mathbf{M}}^{-1}(\widetilde{\mathbf{B}}(\mathbf{q}) \boldsymbol{\tau}-\widetilde{\mathbf{E}}(\mathbf{q}, \dot{\mathbf{q}}) \boldsymbol{v}(t)-\widetilde{\mathbf{C}}(\mathbf{q}, \dot{\mathbf{q}})),
\end{aligned}
$$

where

$$
\begin{aligned}
& \widetilde{\mathbf{M}}=\mathbf{S}^{T} \mathbf{M S}=\left[\begin{array}{cc}
m & 0 \\
0 & m x_{I C R}^{2}+I
\end{array}\right], \quad \widetilde{\mathbf{E}}(\mathbf{q}, \dot{\mathbf{q}})=\mathbf{S}^{T} \mathbf{M} \dot{\mathbf{S}}=\left[\begin{array}{cc}
0 & m x_{I C R} \dot{\theta} \\
-m x_{I C R} \dot{\theta} & m x_{I C R} \dot{x}_{I C R}
\end{array}\right], \\
& \widetilde{\mathbf{C}}(\mathbf{q}, \dot{\mathbf{q}})=\mathbf{S}^{T} \mathbf{C}=\left[\begin{array}{c}
2 R_{l} \\
F_{y} x_{I C R}+M_{r}
\end{array}\right], \quad \widetilde{\mathbf{B}}(\mathbf{q})=\mathbf{S}^{T} \mathbf{B}=\frac{1}{r}\left[\begin{array}{cc}
1 & 1 \\
-b / 2 & b / 2
\end{array}\right] .
\end{aligned}
$$

\section{System Overview}

The introduced kinematics and dynamics of the tracked vehicle are used to design the control system and the system identification framework. This section aims to provide a high-level overview of the overall system design. In the beginning, a block diagram of the system is presented, and a brief description of each subsystem and their interfaces is provided. Furthermore, the measurement system is described.

\subsection{Block Diagram of the System}

The overall system consists of five sub-modules representing different functionalities. Figure 6 shows the block diagram of the system.

The base element of the whole structure is the tracked vehicle platform represented in the diagram with the vehicle model block. The model is described in Sections 2-4. 


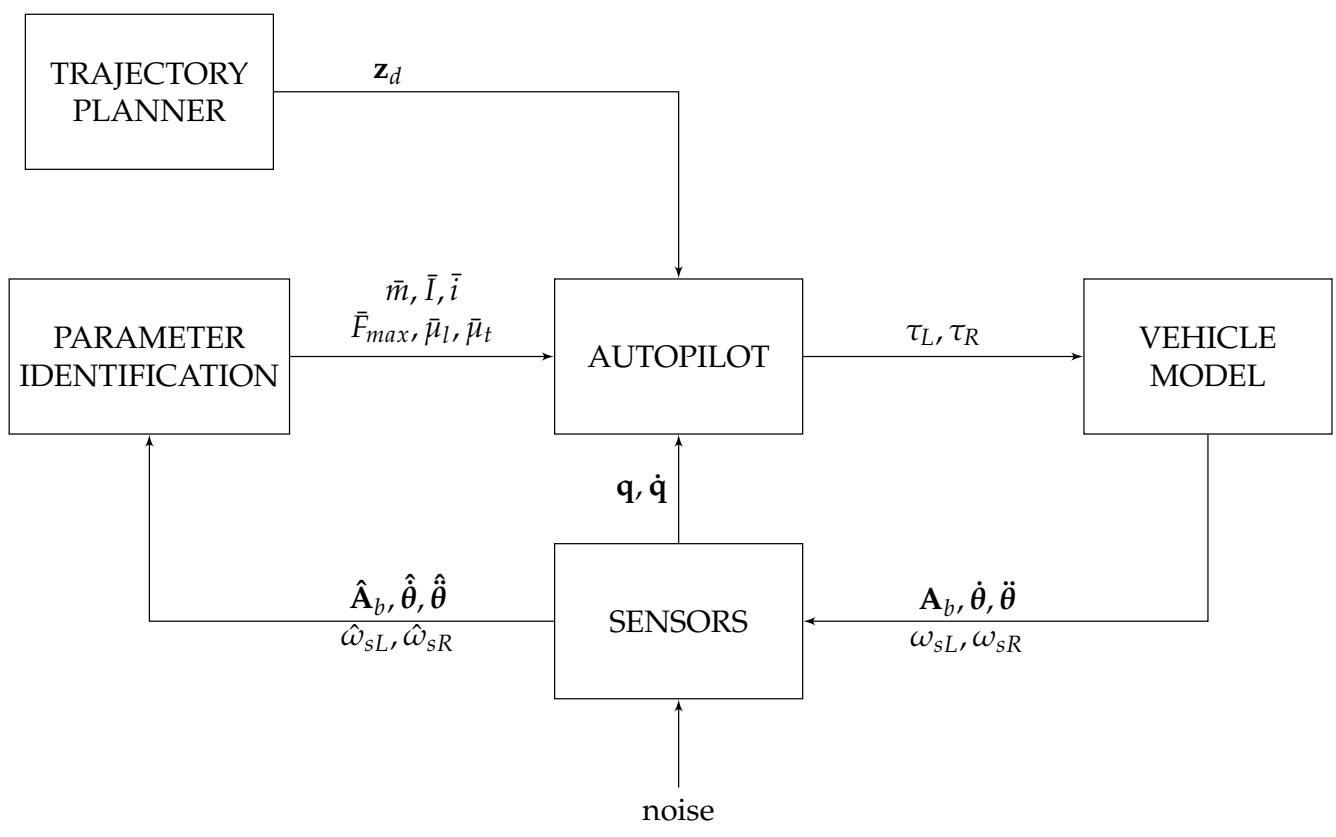

Figure 6. Block diagram of the tracked vehicle system.

The vehicle is controlled with an autopilot sending computed torque demand to the motor controller. The autopilot obtains a reference trajectory from the trajectory planer, which, in this research, was composed of a couple of the predefined trajectories. At the same time, information about the current vehicle state is fed to the autopilot from the onboard sensors. The autopilot computes the control inputs based on the difference between the desired trajectory and the actual state of the vehicle.

Concurrently, measurements obtained with sensors are provided to the parameter identification framework. The updated values of the system parameters are estimated and passed to the autopilot to improve the trajectory tracking performance of the platform.

The sensors block function is introduced to mimic the behavior of real sensors, including dynamics and noise, providing more realistic simulations. More detailed discussion on the sensors can be found in the following section.

\subsection{Measurements and Sensors}

The control scheme, as well as the identification process, requires knowledge about the current system state. Such information can be obtained through measurement of observable states. Today, there is a wide choice of measurement instrumentation in the market, varying in quality and price. In this research, the authors aimed to choose the most affordable solution possible.

\subsubsection{Position, Velocity and Acceleration}

One of the most popular devices utilized to obtain information about the vehicle motion is the inertial measurement unit (IMU), which combines multiple sensors, such as accelerometers, gyroscopes, and magnetometers. At the output, IMU provides the vehicle acceleration and rotation rate, that can be further integrated to obtain the velocities and position in space. Although IMU measurements are quite accurate and provide information at high frequency, they suffer from accumulating error in time. IMUs are prone to the influence of noise (needed signals (speed and acceleration) for pose computation are derivations of the base signal), which accelerates the error accumulation. Odometry as an inertial navigation system (INS) that uses IMU measurements, for example, accumulates the pose error with the square of the traversed distance.

To improve the accuracy of IMU measurements, an inertial navigation system (INS) combined with GPS technology can be introduced. GPS measurements are generally less accurate and sampled at lower frequencies. However, it is quite a common strategy to 
combine data from different sources each having its errors and sampling frequencies to get better estimations [41]. In GPS/INS, the data obtained from IMU is fused with the GPS measurements using Kalman Filter to correct the IMU bias and increase the overall system accuracy [42].

Identification of the moment of inertia requires information on the vehicle angular acceleration. This can be obtained with two accelerometers mounted at different distances from the rotation center [43]. Let us assume that the accelerometers are placed at distances $r_{1}$ and $r_{2}$ from the vehicle center of rotation, respectively (see Figure 7), and $a_{y 1}$ and $a_{y 2}$ denote the tangential accelerations measured with the accelerometers. Distance between the devices can be computed as $D=r_{2}-r_{1}$, and then the angular acceleration is given by $\ddot{\theta}=\left(a_{y 1}-a_{y 2}\right) / D$.

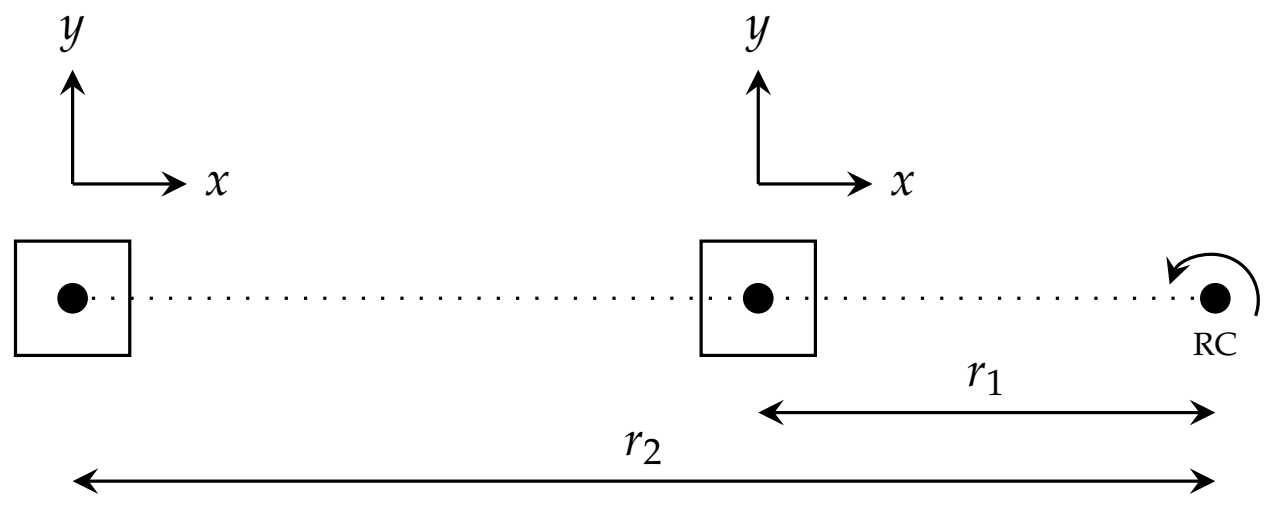

Figure 7. Positioning of the accelerometers for rotational measurements.

In the simulation, the INS/GPS sensors are modeled with the IMU and INS/GPS objects available with MATLAB $^{\circledR}$ Sensor Fusion and Tracking Toolbox. INS/GPS sensor noise is modeled as a white noise process. For the simulations conducted in this research, the following INS/GPS parameters are set:

- yaw accuracy: $\sigma=1 \mathrm{deg}$,

- position accuracy: $\sigma=1 \mathrm{~m}$, and

- velocity accuracy: $\sigma=0.1 \mathrm{~m} / \mathrm{s}$.

For the IMU sensor, accelerometer and gyroscope performance is also specified. Values of the parameters employed in the simulation were found in the datasheets of the IMU sensors available in the market

- gyro bias: $0.01 \mathrm{rad} / \mathrm{s}$,

- accelerometer bias: $0.002 \mathrm{~m} / \mathrm{s}^{2}$, and

- axis cross-coupling: 0.001 .

Assuming that $s_{\text {real }}$ is the real signal value and $s_{\text {meas }}$ is the measured value, then the measurement error is given by $s=s_{\text {real }}-s_{\text {meas }}$.

\subsubsection{Rotational Velocity of the Sprockets and Motor Torque}

Another quantity that needs to be measured in the system is the rotational speed of the tracks. There are two possible ways of obtaining this information. First, the shaft decoders can be utilized. The second approach is to estimate the rotation rate from the motor current measurement (applicable when the electric motors actuate the system). Torque and, consequently, rotational speed measurement can be performed with sensors available on the market.

In the simulation environment, sprocket rotational speed, as well as torque measurements, are obtained by introducing the additive white noise to the computed signal value. In Simulink, the noise is incorporated into the signal with AWGN Channel block that can be found in Communications Toolbox. In this study, we defined the variance of white noise 
added to the input signals. For both torque and sprocket rotational speed, the variance was set to $\sigma^{2}=(0.1)^{2}$.

\section{Control}

In this chapter, the derivation of the control scheme for the tracked vehicle is described. Our control objective is to accurately guide the vehicle along the desired trajectory. Below, a thorough description of the employed method is presented.

\subsection{Static State Feedback}

The tracked vehicle is a nonlinear system, therefore, to be able to apply linear control techniques, it is vital to algebraically transform a nonlinear dynamics into a linear form.

First, nonlinear static state feedback is applied to compensate for the system dynamics. From Equation (28), we can obtain the expression for torque:

$$
\boldsymbol{\tau}=\widetilde{\mathbf{B}}^{-1}(\mathbf{q})(\widetilde{\mathbf{M}} \dot{\boldsymbol{v}}+\widetilde{\mathbf{E}}(\mathbf{q}, \dot{\mathbf{q}}) \boldsymbol{v}(t)+\widetilde{\mathbf{C}}(\mathbf{q}, \dot{\mathbf{q}}))
$$

Following Reference [11], we choose the new control variable $u=\left[\begin{array}{ll}u_{1} & u_{2}\end{array}\right]^{T}$ and transform the torque control signal so that input $u$ becomes proportional to the system acceleration response $\dot{\boldsymbol{v}}$, namely

$$
\boldsymbol{\tau}=\widetilde{\mathbf{B}}^{-1}(\widetilde{\mathbf{M}} \mathbf{u}+\widetilde{\mathbf{E}} \boldsymbol{v}+\widetilde{\mathbf{C}}),
$$

and results in a torque control signal of the following explicit form:

$$
\left[\begin{array}{c}
\tau_{1} \\
\tau_{2}
\end{array}\right]=\left[\begin{array}{c}
\frac{r}{2}\left(m u_{1}+m v_{2}^{2}+2 R_{l}\right) \\
+\frac{r}{b}\left(\left(m x_{I C R}^{2}+I\right) u_{2}-m x_{I C R} v_{1} v_{2}+m x_{I C R} \dot{x}_{I C R} v_{2}+F_{y}+M_{r}\right) \\
\frac{r}{2}\left(m u_{1}+m v_{2}^{2}+2 R_{l}\right) \\
-\frac{r}{b}\left(\left(m x_{I C R}^{2}+I\right) u_{2}-m x_{I C R} v_{1} v_{2}+m x_{I C R} \dot{x}_{I C R} v_{2}+F_{y}+M_{r}\right)
\end{array}\right] .
$$

Thus, a second-order kinematic model is obtained:

$$
\begin{aligned}
& \dot{\mathbf{q}}=\mathbf{S} v, \\
& \dot{v}=\mathbf{u},
\end{aligned}
$$

which gives:

$$
\begin{aligned}
& \dot{X}=v_{1} \cos \theta+v_{2} x_{I C R} \sin \theta, \\
& \dot{Y}=v_{1} \sin \theta-v_{2} x_{I C R} \cos \theta, \\
& \dot{\theta}=v_{2}, \\
& \dot{v}_{1}=u_{1}, \\
& \dot{v}_{2}=u_{2} .
\end{aligned}
$$

\subsection{Input-Output Linearization}

In the next step, the dynamic state feedback is applied so that the system becomes input-output decoupled.

Let us consider a smooth affine nonlinear system:

$$
\begin{aligned}
& \dot{x}=f(x)+G(x) u, \\
& z=h(x),
\end{aligned}
$$


with $x, u$, and $z$ being the system state, input, and output, respectively. Moreover, we assume that the number of inputs and outputs is equal. In case of linearization via static feedback, we seek for the control law of the following form [18]

$$
u=a(x)+B(x) r,
$$

where $r$ is an external auxiliary input of the same size as $u$, and $B(x)$ is non-singular decoupling matrix. Sometimes it is not possible to solve the problem by means of static feedback. In such case, a dynamic feedback compensator might be successfully utilized: [18]

$$
\begin{aligned}
& u=a(x, \xi)+B(x, \xi) r \\
& \dot{\xi}=c(x, \xi)+D(x, \xi) r
\end{aligned}
$$

where $\xi$ is the state compensator.

In the case of the tracked vehicle, a new set of linearizing outputs needs to be chosen for the decoupling matrix to be non-singular [11]. Hence, we choose to observe the position of a point $D$ located on the x-axis at distance $d$ from the body-frame origin. Therefore, the coordinates of the point $D$ in the inertial frame are given by

$$
\mathbf{z}=\left[\begin{array}{l}
X+d \cos \theta \\
Y+d \sin \theta
\end{array}\right] .
$$

Then, the dynamic extension is introduced on the input $u_{1}$

$$
\begin{aligned}
& u_{1}=\xi, \\
& \dot{\xi}=\eta_{1}, \\
& u_{2}=\eta_{2}
\end{aligned}
$$

with $\eta_{1}, \eta_{2}$ being the new control inputs.

In the input-output decoupling algorithm, the output $\mathbf{z}$ is differentiated until the new input $\eta$ appears explicitly in the equations

$$
\begin{aligned}
\dot{\mathbf{z}} & =\left[\begin{array}{l}
\dot{X}-d s \theta \dot{\theta} \\
\dot{Y}+d c \theta \dot{\theta}
\end{array}\right]=\left[\begin{array}{l}
v_{1} c \theta+v_{2} x_{I C R} s \theta-d s \theta v_{2} \\
v_{1} s \theta-v_{2} x_{I C R} c \theta+d c \theta v_{2}
\end{array}\right]=\left[\begin{array}{l}
v_{1} c \theta \\
v_{1} s \theta
\end{array}\right], \\
\ddot{\mathbf{z}} & =\left[\begin{array}{l}
\dot{v}_{1} c \theta-v_{1} s \theta \dot{\theta} \\
\dot{v}_{1} s \theta+v_{1} c \theta \dot{\theta}
\end{array}\right]=\left[\begin{array}{l}
u_{1} c \theta-v_{1} v_{2} s \theta \\
u_{1} s \theta+v_{1} v_{2} c \theta
\end{array}\right], \\
\dddot{\mathbf{z}} & =\left[\begin{array}{l}
\dot{u}_{1} c \theta-u_{1} v_{2} s \theta-\dot{v}_{1} v_{2} s \theta-v_{1} \dot{v}_{2} s \theta-v_{1} v_{2} c \theta \dot{\theta} \\
\dot{u}_{1} s \theta+u_{1} v_{2} c \theta+\dot{v}_{1} v_{2} c \theta+v_{1} \dot{v}_{2} c \theta-v_{1} v_{2} s \theta \dot{\theta}
\end{array}\right] \\
& =\left[\begin{array}{l}
\eta_{1} c \theta-2 \xi v_{2} s \theta-v_{1} \eta_{2} s \theta-v_{1} v_{2}^{2} c \theta \\
\eta_{1} s \theta+2 \xi \\
v_{2} c \theta+v_{1} \eta_{2} c \theta-v_{1} v_{2}^{2} s \theta
\end{array}\right] .
\end{aligned}
$$

Subsequently, Equation (32c) can be rearranged to a more convenient form:

$$
\begin{aligned}
\dddot{\mathbf{z}} & =\left[\begin{array}{cc}
c \theta & -v_{1} s \theta \\
s \theta & v_{1} c \theta
\end{array}\right] \boldsymbol{\eta}+\left[\begin{array}{c}
-2 \xi v_{2} s \theta-v_{1} v_{2}^{2} c \theta \\
2 \xi v_{2} c \theta-v_{1} v_{2}^{2} s \theta
\end{array}\right] \\
& =\alpha(\mathbf{q}, \boldsymbol{v}) \boldsymbol{\eta}+\beta(\mathbf{q}, \boldsymbol{v}, \boldsymbol{\xi}) .
\end{aligned}
$$

Further, we rearrange the above equation to obtain the control law

$$
\boldsymbol{\eta}=\alpha^{-1}(\mathbf{q}, \boldsymbol{v})[\mathbf{r}-\beta(\mathbf{q}, \boldsymbol{v}, \xi)],
$$

where we introduce the trajectory jerk reference $\mathbf{r}=\dddot{\mathbf{z}}$. From the determinant of $\alpha$

$$
\operatorname{det}[\alpha(\mathbf{q}, \boldsymbol{v})]=v_{1},
$$


we can conclude that the decoupling matrix is non-singular apart from the situation when the linear speed of the vehicle is zero, i.e., $v_{1}=0$, which does not negatively influence the tracking performance for the persistent trajectories.

Combining Equation (31) with Equation (33) yields the final form of fully linearizing, input-output decoupling controller:

$$
\begin{aligned}
& \dot{\xi}=r_{1} \cos \theta+r_{2} \sin \theta+v_{1} v_{2}^{2} \\
& u_{1}=\xi \\
& u_{2}=\frac{1}{v_{1}}\left(-r_{1} \sin \theta+r_{2} \cos \theta\right)-2 \xi \frac{v_{2}}{v_{1}}
\end{aligned}
$$

\subsection{Exponentially Stabilizing Feedback for Tracking}

Once full-state linearization is obtained, the control design can be completed with a globally stabilizing feedback for the desired smooth trajectory $z_{d}(t)$ [18]:

$$
r_{i}=\dddot{z}_{d i}+k_{a i}\left(\ddot{z}_{d i}-\ddot{z}_{i}\right)+k_{v i}\left(\dot{z}_{d i}-\dot{z}_{i}\right)+k_{p i}\left(z_{d i}-z_{i}\right), \quad i=\{1,2\} .
$$

The feedback gains are chosen so that the polynomials,

$$
\lambda^{3}+k_{a i} \lambda^{2}+k_{v i} \lambda+k_{p i}
$$

are Hurwitz polynomials, and $\mathbf{z}, \dot{\mathbf{z}}, \ddot{\mathbf{z}}$ can be computed with Equations (30) and (32a-c). Thus, we obtained the control scheme that results in the following open-loop transfer function of the system [11]

$$
F(s)=C(s) P(s)=\frac{k_{a} s^{2}+k_{v} s+k_{p}}{s^{3}} .
$$

The block diagram of the proposed controller is illustrated in Figure 8.

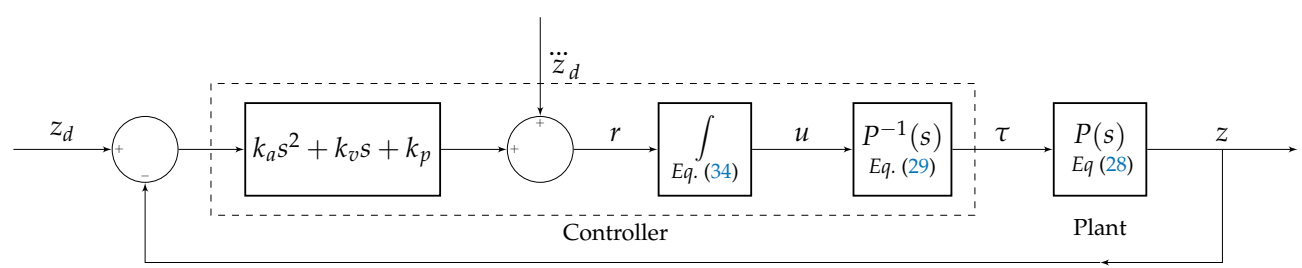

Figure 8. Block diagram of the trajectory tracking control system.

It should be noted that Hurwitz polynomials guarantee the stability of the developed controller. In the case of perfect knowledge of the vehicle dynamics model, the proposed control law perfectly performs trajectory tracking. In case of uncertain model dynamics parameters, the vehicle closed-loop dynamics will be nonlinear and coupled. The effect of the estimation error is a torque disturbance that could be rejected by the controller augmented with adaptive capabilities and by avoiding unfeasible commands via proper path planning.

\section{Parameter Identification}

The vehicle mass, inertia, and terrain behavior can vary due to mission operations or moving through unknown terrain. Imprecise knowledge of the vehicle model can lead to poor control performance. However, model parameters can be estimated on-line and provided to the controller and / or the trajectory planner to improve the system performance.

Selection of the model structure and parametrization are crucial steps of the system identification. Within this research, the models of ground vehicle dynamics and vehicleterrain interaction described above are used for parametrization. In particular, values of the vehicle mass, inertia, and also parameters of the vehicle-terrain interaction, such as slip, resistance coefficients, cohesion $F_{\max }$, and shear deformation modulus $K$, are estimated on 
the fly. Analysis of the model structure enabled selection of the proper estimation technique for each of the mentioned parameters.

\subsection{Estimations of Mass, Inertia and Motion Resistance}

The EF RLS is a common approach for estimation of mass, inertia, and road grade based on the model of longitudinal and rotational dynamics of the ground vehicle [23,24], and it was applied to estimate mass, inertia, and motion resistance coefficients in the current research.

Let us first give a brief overview of the system identification methods used in the current research.

\subsubsection{Recursive Least Squares with Exponential Forgetting}

Recursive Least Squares with Exponential Forgetting (RLS) algorithm is the most common approach since it is simple and can be applied to a large variety of on-line estimation problems.

Assume that a process is generated by the following model:

$$
y(t)=\boldsymbol{\phi}^{T}(t) \boldsymbol{\vartheta}+\varepsilon(t),
$$

where $y$ is the observed variable, $\phi$ is the vector of known functions, $\varepsilon$ is the measurement noise, and $\vartheta$ is the vector of unknown parameters.

The estimation $\hat{\vartheta}(k)$ of unknown parameter vector $\vartheta$ at a step $k$ can be obtained recursively using the following equations $[23,44]$ :

$$
\begin{aligned}
\hat{\boldsymbol{\vartheta}}(k) & =\hat{\boldsymbol{\vartheta}}(k)+\boldsymbol{K}(k) \varepsilon(k), \\
\varepsilon(k) & =y(k)-\boldsymbol{\phi}^{T}(k) \hat{\boldsymbol{\vartheta}}(k-1), \\
\boldsymbol{K}(k) & =\frac{P(k-1) \boldsymbol{\phi}(k)}{\lambda+\boldsymbol{\phi}^{T}(k) P(k-1) \boldsymbol{\phi}(k)}, \\
P(k) & =\frac{1}{\lambda}\left[P(k-1)-\frac{P(k-1) \boldsymbol{\phi}(k) \boldsymbol{\phi}^{T}(k) P(k-1)}{\lambda+\boldsymbol{\phi}^{T}(k) P(k-1) \boldsymbol{\phi}(k)}\right],
\end{aligned}
$$

where $\boldsymbol{K}(k)$ is the adaptation gain vector, and $P(k)$ is the covariance matrix.

\subsubsection{Identification Parametrization}

Following the common way, here, we estimate the mass and longitudinal resistance coefficient based on the longitudinal dynamics. If the torque converter and the driveline are fully engaged, it can be assumed that all the torque from the engine is fully passed to the track. Another assumption made here is that the service brake is not applied during identification procedures, and the friction brake force is not generated. Further, we assume that there is no slip, which is an acceptable assumption, for the most part. It is also assumed that the aerodynamic loads can be neglected since the vehicle travels with a very low speed. Under these assumptions, the longitudinal dynamics can be presented in the following simplified form:

$$
m \ddot{X}=\frac{\tau_{L}+\tau_{R}}{r}-2 R_{l} .
$$

We want to rearrange the above equation and represent it in the form of Equation (36). Note that $R_{l}$ is also dependent on $m$, as well as $\mu_{l}$. We obtain

$$
\ddot{X}=\frac{\tau_{L}+\tau_{R}}{r} \frac{1}{m}-g \mu_{l},
$$

where $y=\ddot{X}, \boldsymbol{\phi}=\left[\begin{array}{ll}\left(\tau_{L}+\tau_{R}\right) / r & -g\end{array}\right]^{T}$ and $\hat{\vartheta}=\left[\begin{array}{ll}1 / m & \mu_{l}\end{array}\right]^{T}$. 
The inertia and lateral resistance indices can be evaluated from the rotational dynamics model. To simplify parametrization of the identification, let us write the rotational dynamics of the tracked vehicle in the body fixed reference frame in the following way [3]:

$$
(I) \ddot{\theta}=\frac{b\left(\tau_{L}-\tau_{R}\right)}{2 r}-M_{r}
$$

Recalling the expression for $M_{r}$ from Equations (20) and (21) and rearranging the Equation (37) into the form of Equation (36), we can obtain

$$
\frac{b\left(\tau_{L}-\tau_{R}\right)}{2 r}=\ddot{\theta} I-\frac{2 \operatorname{sign}(\dot{\theta}) m g}{l}\left(\frac{l^{2}}{4}-x_{I C R}^{2}\right) \mu_{t},
$$

where

$$
\begin{aligned}
y & =\frac{b\left(\tau_{L}-\tau_{R}\right)}{2 r}, \\
\phi & =\left[\begin{array}{ll}
\ddot{\theta} & \frac{-2 \operatorname{sign}(\dot{\theta}) m g}{l}\left(\frac{l^{2}}{4}-x_{I C R}^{2}\right)
\end{array}\right]^{T}, \\
\vartheta & =\left[\begin{array}{ll}
I & \mu_{t}
\end{array}\right]^{T} .
\end{aligned}
$$

\subsection{Slip Estimation}

As it is discussed earlier, the Unscented Kalman Filter (UKF) demonstrates quite accurate estimation of parameters and can be effectively used for longitudinal slip identification. A brief description of the UKF algorithm is provided in the next subsection, followed by the derivation of state and measurement equations for the tracked vehicle.

\subsubsection{Unscented Kalman Filter}

UKF is an effective technique for estimating the state of a nonlinear dynamic system. Let us assume that a system has the following nonlinear dynamics:

$$
\begin{aligned}
& \mathbf{x}_{k+1}=f\left(\mathbf{x}_{k}, \mathbf{u}_{k}\right)+\mathbf{v}_{k} \\
& \mathbf{z}_{k}=h\left(\mathbf{x}_{k}\right)+\mathbf{w}_{k},
\end{aligned}
$$

where $\mathbf{x}_{k}$ is a process state vector at time $t_{k}, \mathbf{u}_{k}$ is a vector of the control inputs, $\mathbf{z}_{k}$ is a measurement vector, and $f\left(\mathbf{x}_{k}, \mathbf{u}_{k}\right)$ and $h\left(\mathbf{x}_{k}\right)$ are state transition function and measurement function, respectively. Finally, $\mathbf{v}_{k}$ and $\mathbf{w}_{k}$ denote the additive white noise of the covariance determined by $\mathbf{Q}_{k}$ and $\mathbf{R}_{k}$, respectively.

Approximations associated with the linearization process, e.g., in Extended Kalman Filter (EKF), may lead to noticeable errors in the covariance and posterior mean of the transformed random variable, which, subsequently, can cause a sub-optimal performance or even divergence of the filter [45].

The approximation drawbacks described above are eliminated with the UKF, where the prior and posterior mean and covariance are represented by minimal set of weighted samples, so called sigma points. The UKF utilizes an unscented transformation method for calculating the statistics of a random variable which undergoes a nonlinear transformation. In the unscented transform, prior sigma points projection through nonlinear function gives results very close to the real transformed distribution [46]. The applied method for the sigma points derivation is described below.

Let us assume that mean and covariance of the random variable $\mathbf{x}$ are denoted with $\hat{\mathbf{x}}$ and $\mathbf{P}_{\mathbf{x}}$, respectively. The variable is propagated through the nonlinear functions $\mathbf{x}_{k+1}=f\left(\mathbf{x}_{k}\right)$ and $\mathbf{z}_{k}=h\left(\mathbf{x}_{k}\right)$. For this purpose, a matrix $\mathcal{X}$ of sigma points (Equation (38)) is formed, and the corresponding weights $W_{i}$ (Equation (39)) are computed. The number 
of sigma points is defined with the expression: $2 L+1$, where $L$ is dimension of the $\mathbf{x}$ vector [46]:

$$
\begin{aligned}
& \mathcal{X}_{0}=\hat{\mathbf{x}}, \\
& \mathcal{X}_{i}=\hat{\mathbf{x}}+\left(\sqrt{(L+\lambda) \mathbf{P}_{\mathbf{x}}}\right)_{i} \quad i=1, \cdots, L, \\
& \mathcal{X}_{i}=\hat{\mathbf{x}}-\left(\sqrt{(L+\lambda) \mathbf{P}_{\mathbf{x}}}\right)_{i} i=L+1, \cdots, 2 L, \\
& W_{0}^{(m)}=\lambda /(L+\lambda), \\
& W_{0}^{(c)}=\lambda /(L+\lambda)+\left(1-\alpha_{u k f}^{2}+\beta_{u k f}\right), \\
& W_{i}^{(m)}=W_{i}^{(c)}=1 /[2(L+\lambda)] \quad i=1, \cdots, 2 L,
\end{aligned}
$$

where $\lambda=\alpha_{u k f}^{2}\left(L+\kappa_{u k f}\right)$ and $\kappa_{u k f}$ are the scaling parameters, $\alpha_{u k f}$ determines how sigma points are spread around mean, and $\beta_{u k f}=2$ for Gaussian distributions and should be chosen basing on the prior knowledge about the random variable distribution.

Further, points are propagated through the nonlinear functions

$$
\begin{aligned}
\mathcal{X}_{k+1 \mid k}^{i} & =f\left(\mathcal{X}_{k}^{i}, \mathbf{u}_{k}\right), \quad i=0, \cdots, 2 L, \\
\mathcal{Z}_{k+1 \mid k}^{i} & =h\left(\mathcal{X}_{k+1 \mid k}^{i}\right) .
\end{aligned}
$$

Subsequently, the mean and the covariance of $\mathbf{x}$ and $\mathbf{z}$ are computed:

$$
\begin{gathered}
\hat{\mathbf{x}}_{k+1}^{-}=\sum_{i=0}^{2 L} W_{i}^{(m)} \mathcal{X}_{k+1 \mid k^{\prime}}^{i} \\
\hat{\mathbf{z}}_{k+1}^{-}=\sum_{i=0}^{2 L} W_{i}^{(m)} \mathcal{Z}_{k+1 \mid k^{\prime}}^{i} \\
\mathbf{P}_{k+1 \mid k}=\sum_{i=0}^{2 L} W_{i}^{(c)}\left(\mathcal{X}_{k+1 \mid k}^{i}-\hat{\mathbf{x}}_{k+1}^{-}\right)\left(\mathcal{X}_{k+1 \mid k}^{i}-\hat{\mathbf{x}}_{k+1}^{-}\right)^{T}+\mathbf{Q}_{k \prime} \\
\mathbf{P}_{\widetilde{\mathbf{z}} \mathbf{z}}=\sum_{i=0}^{2 L} W_{i}^{(c)}\left(\mathcal{Z}_{k+1 \mid k}^{i}-\hat{\mathbf{z}}_{k+1}^{-}\right)\left(\mathcal{Z}_{k+1 \mid k}^{i}-\hat{\mathbf{z}}_{k+1}^{-}\right)^{T}+\mathbf{R}_{k \prime} \\
\mathbf{P}_{\widetilde{\mathbf{x}} \mathbf{z}}=\sum_{i=0}^{2 L} W_{i}^{(c)}\left(\mathcal{X}_{k+1 \mid k}^{i}-\hat{\mathbf{x}}_{k+1}^{-}\right)\left(\mathcal{Z}_{k+1 \mid k}^{i}-\hat{\mathbf{z}}_{k+1}^{-}\right)^{T} .
\end{gathered}
$$

In the last step, the Kalman gain is computed:

$$
\mathbf{K}_{k+1}=\mathbf{P}_{\widetilde{\mathbf{x}} \mathbf{z}} \mathbf{P}_{\widetilde{\mathbf{z}} \mathbf{\mathbf { z }}}-1,
$$

and the new state and covariance estimate are obtained:

$$
\begin{gathered}
\hat{\mathbf{x}}_{k+1}=\hat{\mathbf{x}}_{k+1}^{-}+\mathbf{K}_{k+1}\left(\mathbf{z}_{k+1}-\hat{\mathbf{z}}_{k+1}^{-}\right), \\
\mathbf{P}_{k+1}=\mathbf{P}_{k+1 \mid k}+\mathbf{K}_{k+1} \mathbf{P}_{\widetilde{\mathbf{z}} \mathbf{z}} \mathbf{K}_{k+1}^{T} .
\end{gathered}
$$

\subsubsection{Identification Parametrization}

The UKF is designed to recover the slip parameters $i_{L}$ and $i_{R}$ from the vehicle states. Therefore, the augmented state vector is formed:

$$
\mathbf{x}=\left[\begin{array}{llllll}
X & Y & \theta & i_{L} & i_{R} & \alpha
\end{array}\right]^{T},
$$


and the vector of estimates $\left[\begin{array}{llllll}\hat{X} & \hat{Y} & \hat{\theta} & \hat{i}_{L} & \hat{i}_{R} & \hat{\alpha}\end{array}\right]^{T}$ is obtained from the available measurements:

$$
\mathbf{z}=\left[\begin{array}{lllll}
X_{m} & Y_{m} & \theta_{m} & \omega_{t m, L} & \omega_{t m, R}
\end{array}\right]^{T},
$$

where $\omega_{t m, L}$ and $\omega_{t m, R}$ are the control inputs. The following process model is adopted from the kinematic model of the tracked vehicle from Equation (3):

$$
\mathbf{x}_{k+1}=\left[\begin{array}{c}
X_{k}+\Delta T \cdot 0.5 r\left[\left(1-i_{L, k}\right) \omega_{t, L}^{k+1}+\left(1-i_{R, k}\right) \omega_{t, R}^{k+1}\right]\left(\cos \theta_{k}-\sin \theta_{k} \tan \alpha_{k}\right) \\
Y_{k}+\Delta T \cdot 0.5 r\left[\left(1-i_{L, k}\right) \omega_{t, L}^{k+1}+\left(1-i_{R, k}\right) \omega_{t, R}^{k+1}\right]\left(\sin \theta_{k}+\cos \theta_{k} \tan \alpha_{k}\right) \\
\theta_{k}+\Delta T \frac{r}{b}\left[-\left(1-i_{L, k}\right) \omega_{t, L}^{k+1}+\left(1-i_{R, k}\right) \omega_{t, R}^{k+1}\right] \\
i_{L, k} \\
i_{R, k} \\
\alpha_{k}
\end{array}\right] .
$$

\subsection{Soil Parameters Estimation}

It was shown previously that Generalized Newton-Raphson (GNR) method identifies unknown soil parameter with a high accuracy and rapid convergence [47]. The GNR is employed in this study for the soil parameters estimation that impact the tractive effort, i.e., cohesion $F_{\max }$ and shear deformation modulus $K$. Below, we provide a brief overview of this method and the parametrization of the identification of soil parameters.

\subsubsection{Generalized Newton-Raphson}

The system equation can then be expressed as a function of the parameter vector $\mathbf{p}$ and the measurement vector $\mathbf{x}_{i}[31]$ :

$$
\begin{array}{r}
f_{1}\left(p_{1}, p_{2}, \cdots, p_{n}, x_{1}\left(t_{1}\right), x_{2}\left(t_{1}\right), \cdots, x_{m}\left(t_{1}\right)\right)=0, \\
f_{2}\left(p_{1}, p_{2}, \cdots, p_{n}, x_{1}\left(t_{2}\right), x_{2}\left(t_{2}\right), \cdots, x_{m}\left(t_{2}\right)\right)=0, \\
\cdots \\
f_{q}\left(p_{1}, p_{2}, \cdots, p_{n}, x_{1}\left(t_{q}\right), x_{2}\left(t_{q}\right), \cdots, x_{m}\left(t_{q}\right)\right)=0 .
\end{array}
$$

In the GNR method, $q$ independent equations are required to find the parameter vector $\mathbf{p}$, where $q>n$

$$
\left[\begin{array}{c}
p_{1} \\
p_{2} \\
\vdots \\
p_{n}
\end{array}\right]_{i+1} \approx\left[\begin{array}{c}
p_{1} \\
p_{2} \\
\vdots \\
p_{n}
\end{array}\right]_{i}-\left[\begin{array}{ccc}
\frac{\partial f_{1}}{\partial p_{1}} & \cdots & \frac{\partial f_{1}}{\partial p_{n}} \\
\frac{\partial f_{2}}{\partial p_{1}} & \cdots & \frac{\partial f_{2}}{\partial p_{n}} \\
\vdots & \ddots & \vdots \\
\frac{\partial f_{q}}{\partial p_{1}} & \cdots & \frac{\partial f_{q}}{\partial p_{n}}
\end{array}\right]_{i}^{-1}\left[\begin{array}{c}
f_{1} \\
f_{2} \\
\vdots \\
f_{q}
\end{array}\right]_{i}
$$

The above system of equation is solved recursively until the convergence condition is met.

The main advantage of GNR algorithm is robustness and fast convergence. However, an initial guess of the parameter can influence the convergence rate. Especially, if the initial derivative of a function is close to zero, the convergence speed is low [31]. The advantage of the GNR method over classic Newton-Raphson method is that the former is considered more robust to the measurement noise due to the higher number of samples included in the equation [48].

\subsubsection{Identification Parametrization}

To obtain the estimation model, we need to consider a function in the form

$$
f(\mathbf{p}, \mathbf{x})=0 .
$$


For the tractive force expressed as in Equation (7), we have:

$$
f\left(F_{\max }, K, \tau, i\right)=0,
$$

where: $\mathbf{p}=\left[\begin{array}{ll}F_{\max } & K\end{array}\right]^{T}$ is the vector of parameters to be identified, and $\mathbf{x}=\left[\begin{array}{ll}\tau & i\end{array}\right]^{T}$ is the measurement vector, where $\tau$ is the total torque produced by the motors, and $i$ is the slip. Explicitly, Equation (43) yields:

$$
F_{\max }\left(1-\frac{K}{i l}\left(1-e^{-i l / K}\right)\right)-\frac{\tau}{r}=0,
$$

and Jacobian elements can be obtained with the following expressions:

$$
\begin{aligned}
\frac{\partial f}{\partial F_{\max }} & =1-\frac{K}{i l}\left(1-e^{-i l / K}\right), \\
\frac{\partial f}{\partial K} & =\frac{F_{\max }}{K i l}\left(K\left(1-e^{i l / K}\right)+i l\right) e^{-i l / K} .
\end{aligned}
$$

During the identification process, the algorithm considers $q$ time samples of the measurements to find the solution for Equation (43) using the following equation:

$$
\left[\begin{array}{c}
F_{\max } \\
K
\end{array}\right]_{i+1} \approx\left[\begin{array}{c}
F_{\max } \\
K
\end{array}\right]_{i}-\left[\begin{array}{ll}
\frac{\partial f}{\partial F_{\max }} & \frac{\partial f}{\partial K}
\end{array}\right]_{i}^{-1} f_{i}
$$

\section{Results}

This section presents the simulation outcomes and discussion on the obtained results. The system model was created in MATLAB ${ }^{\circledR} /$ Simulink environment. In the first part of this section, validation of the control system performance is provided. Next, system response to parameters uncertainties is investigated. In the closing part of this section, identification algorithms implemented in the system are evaluated.

Table 1 provides information on the vehicle parameters adopted in the simulation. Default soil parameters are included in Table 2 and are used throughout all the simulations unless stated otherwise.

Table 1. Vehicle parameters.

\begin{tabular}{ccc}
\hline Parameter & Value & Unit \\
\hline Mass, $m$ & 1450 & $\mathrm{~kg}$ \\
Inertia, $I$ & 1180 & $\mathrm{~kg} \cdot \mathrm{m}^{2}$ \\
Tread, $b$ & 1.7 & $\mathrm{~m}$ \\
Track width, $w$ & 0.3 & $\mathrm{~m}$ \\
Track contact length, $l$ & 2 & $\mathrm{~m}$ \\
Sprocket radius, $r$ & 0.3 & $\mathrm{~m}$ \\
Gear ratio, $n$ & $1 / 380$ & \\
\hline
\end{tabular}

Table 2. Soil parameters for heavy clay [5].

\begin{tabular}{ccc}
\hline Parameter & Value & Unit \\
\hline Cohesion, $c$ & 70 & $\mathrm{kPa}$ \\
Angle of internal shearing resistance, $\Phi$ & 38.4 & $\mathrm{deg}$ \\
Shear deformation parameter, $K$ & 0.02 & $\mathrm{~m}$ \\
Coefficient of longitudinal resistance, $\mu_{l}$ & 0.6 & \\
Coefficient of lateral resistance, $\mu_{t}$ & 0.8 & \\
\hline
\end{tabular}


Results presented in this chapter were obtained with a fixed-step solver with the time step $d t=0.01 \mathrm{~s}$. Differential equations were solved with ODE4 (Runge-Kutta) algorithm.

\subsection{Validation of the Control System}

At the beginning, the control system behavior was investigated. Controller gains were chosen experimentally with regard to the stability condition Equation (35). Final values of the feedback gains can be found in Table 3 .

Table 3. Final values of the controller gains.

\begin{tabular}{cc}
\hline Gain & Final Value \\
\hline$k_{p}$ & 50 \\
$k_{v}$ & 70 \\
$k_{a}$ & 15 \\
\hline
\end{tabular}

In the first test, all model parameters are assumed to be known, and the input signals represent the ideal values of the system states.

Figure 9 shows the system response to the circular and straight line trajectory demand.
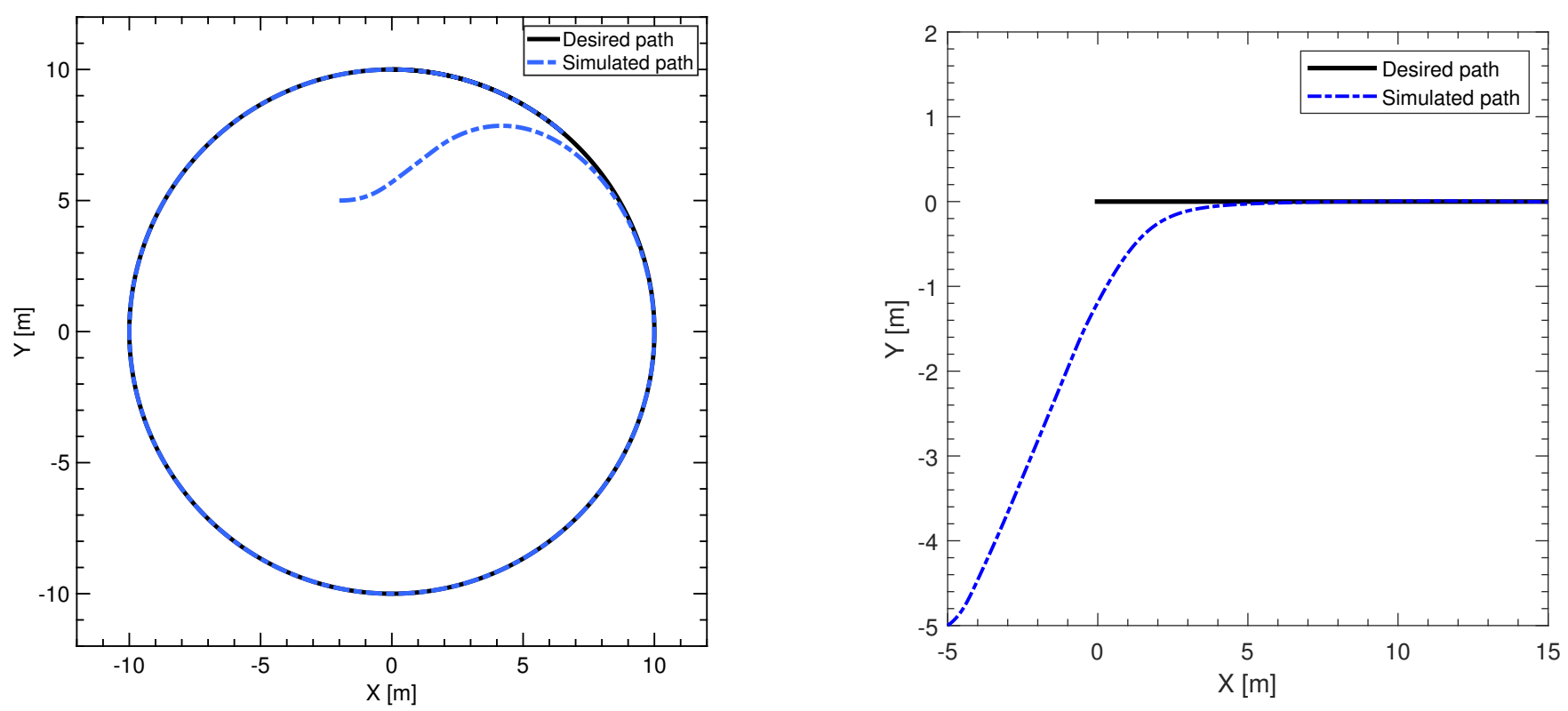

Figure 9. Trajectory following tests: circular (left) and straight (right) path.

From Figure 10, one can see that once on path, the vehicle travels with constant linear and angular velocities that demonstrated on the left and right subplots, respectively.
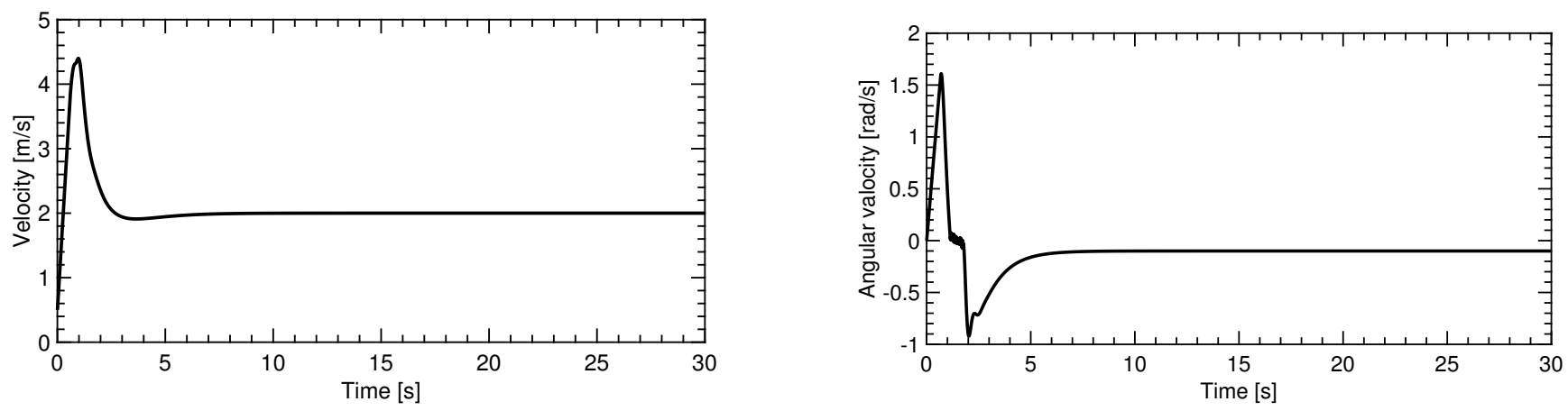

Figure 10. Trajectory following test: forward (left) and rotational (right) velocity. 
According to the results obtained for the circular trajectory (shown as an example), it can be observed that the controller successfully guides the vehicle to the desired path and then continues following the path with no position, velocity, and acceleration error, which is shown in Figure 11.

The behavior of the system parameters, such as torque, sprocket rotational velocity, and slip of the track, is also of interest. These parameters are shown in Figure 12. One can observe that, as soon the vehicle reaches the constant forward and rotational velocities, all the parameters remain of constant magnitudes. Constant turning rate is achieved due to the fixed difference between the left and the right sprocket angular velocities-approximately $1 \mathrm{rad} / \mathrm{s}$. Additionally, one can observe in Figure 12 that the slip of the tracks is directly related to the sprocket torque, as slip characteristics have a similar shape to those obtained for the torque.
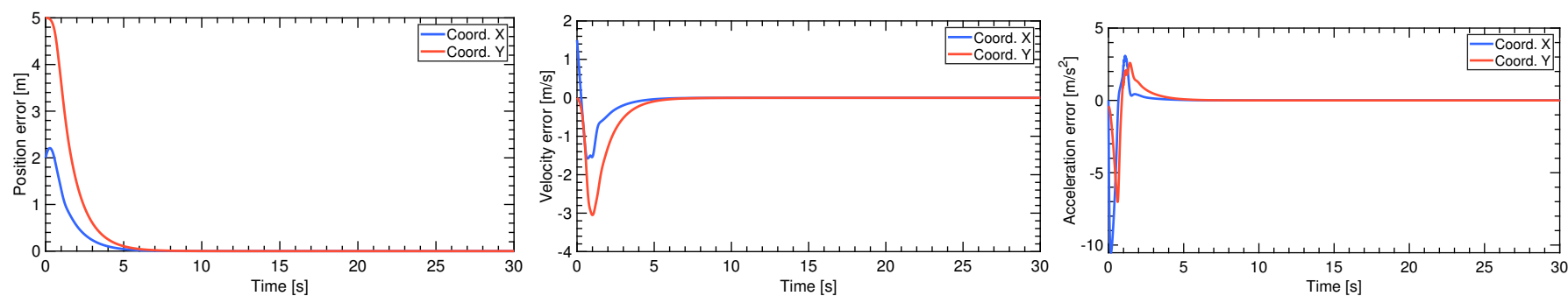

Figure 11. Trajectory following test: position (left), velocity (middle), acceleration (right) errors.
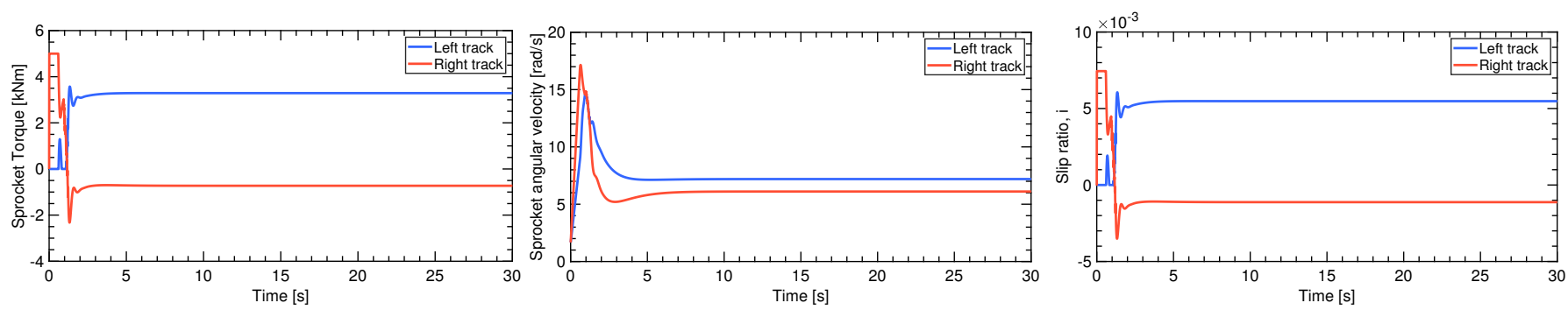

Figure 12. Trajectory following test: torque behavior (left), sprocket rotational velocity (middle), slip of the track (right).

\subsection{System Behavior under Parameter Uncertainties}

In this section, the closed-loop behavior is analyzed under unknown uncertainties introduced in the tracked vehicle dynamics. Different scenarios are considered: in the first scenario, the uncertainties brought in the mass and inertia; in the second scenario, the uncertainties are in the longitudinal and lateral resistance coefficients; and, in the last considered scenario, the uncertainties are introduced in the soil parameters.

\subsubsection{Incorrect Mass and Inertia of the Vehicle}

In the first scenario, mass and inertia of the vehicle used in the controller for torque command calculation differ from the actual values. Figure 13 shows the tracking performance of the controller for the circular and the straight path tests.

The mass provided to the controller is $55 \%$ of the true vehicle mass $m$, and moment I is $110 \%$ of the actual value. For true values, take a look at Table 1 . The introduced uncertainty is quite big, which is purposely caused to obtain a clear view of uncertainty impact produced on the system behavior.

Figure 14 demonstrates the velocity tracking behavior of the controller.

A more detailed look into the position, velocity, and acceleration errors is given in Figure 15. According to these results, one can conclude that the inaccurate knowledge of mass and inertia causes the inaccurate trajectory tracking, but the controller performance remains at the acceptable level. 

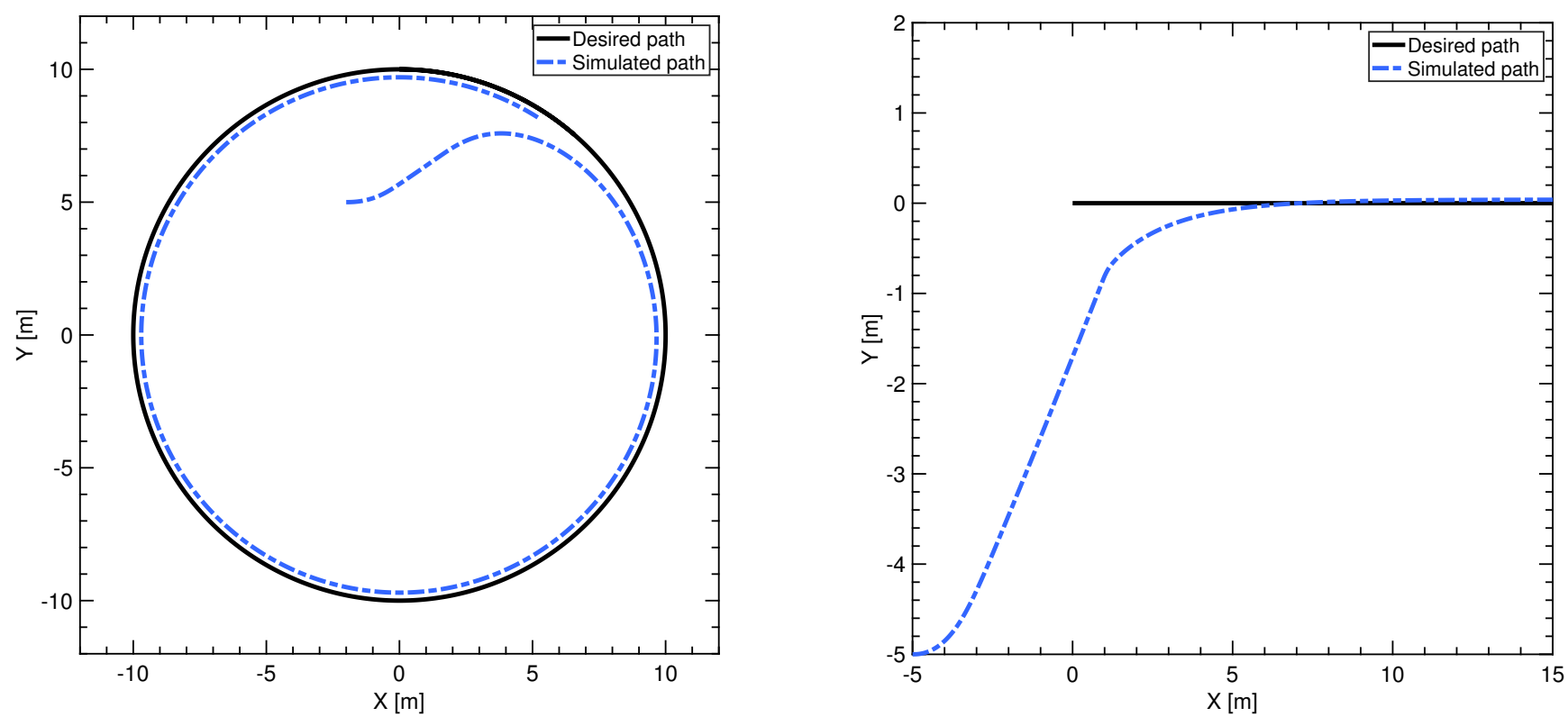

Figure 13. Trajectory following test in case of incorrect knowledge of incorrect mass and inertia: circular (left) and straight paths (right).
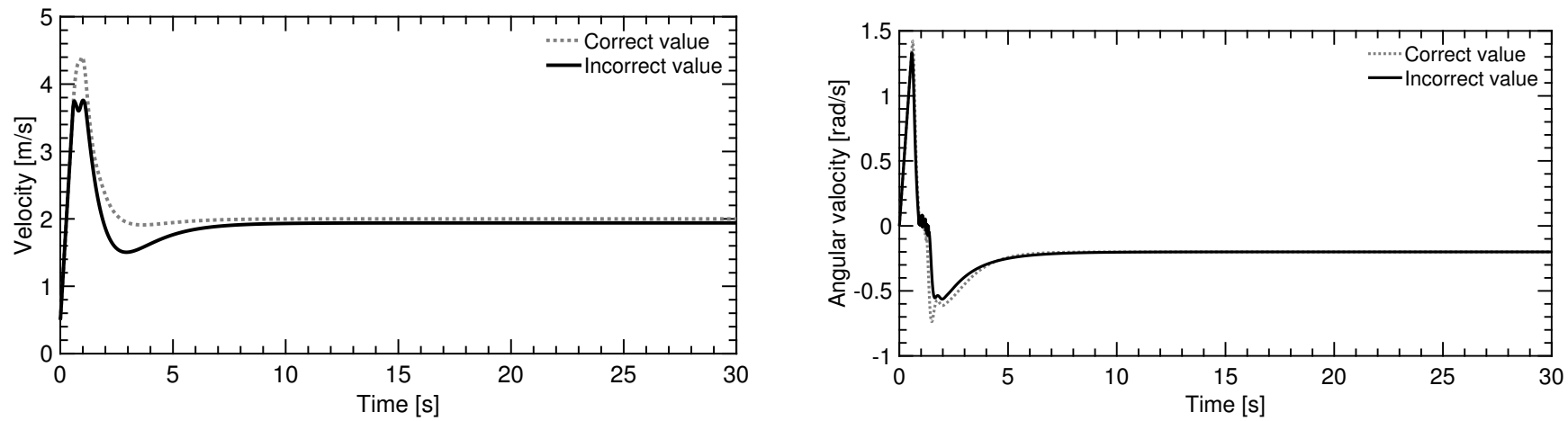

Figure 14. Trajectory following test in case of incorrect knowledge of incorrect mass and inertia: instantaneous forward velocity (left) and rotational velocity(right).
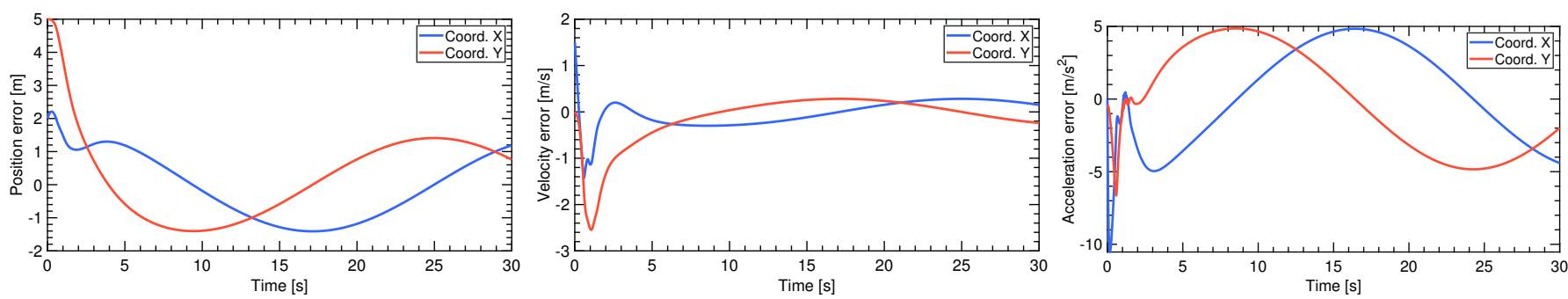

Figure 15. Trajectory following test in case of incorrect knowledge of incorrect mass and inertia: position (left), velocity (middle), acceleration (right) errors.

The torque, sprocket rotational velocity, and the slip of the track produced by the controller designed for the uncertain system is demonstrated in Figure 16, together with the results for a controller designed for the system without uncertainties. The behavior is quite similar, with the only difference in the sprocket rotational velocity during the initial stage (first $10 \mathrm{~s}$ ).

To separate the effects produced by the presence of uncertainty in mass and inertia, the root mean square error (RMSE) of the vehicle trajectory for various levels of uncertainty in the mass and the inertia values as compared to the trajectory obtained for the correct 
model is calculated and plotted in Figure 17. These results manifest that inaccuracies in mass estimation have a greater impact on the trajectory tracking capabilities of the vehicle than the moment of inertia.
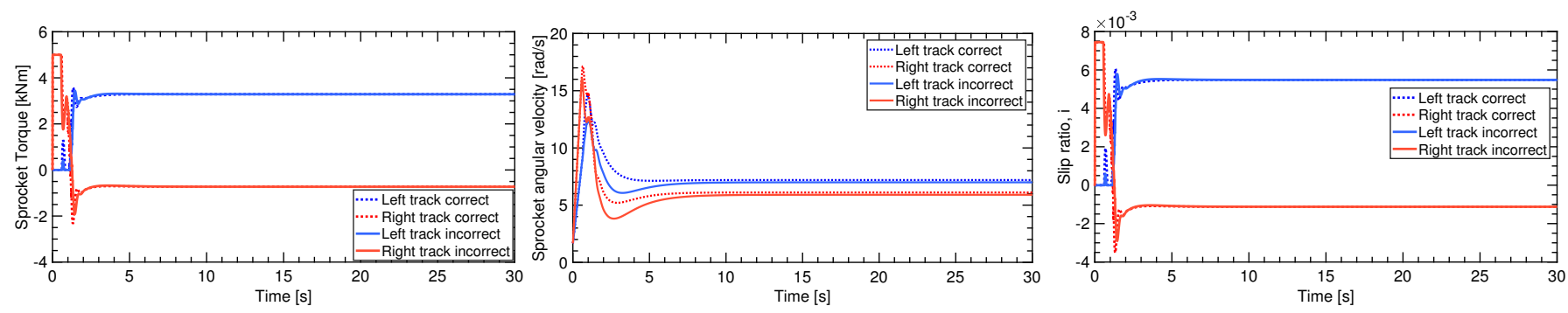

Figure 16. Trajectory following test in case of incorrect knowledge of incorrect mass and inertia: torque behavior (left), sprocket rotational velocity (middle), slip of the track (right).

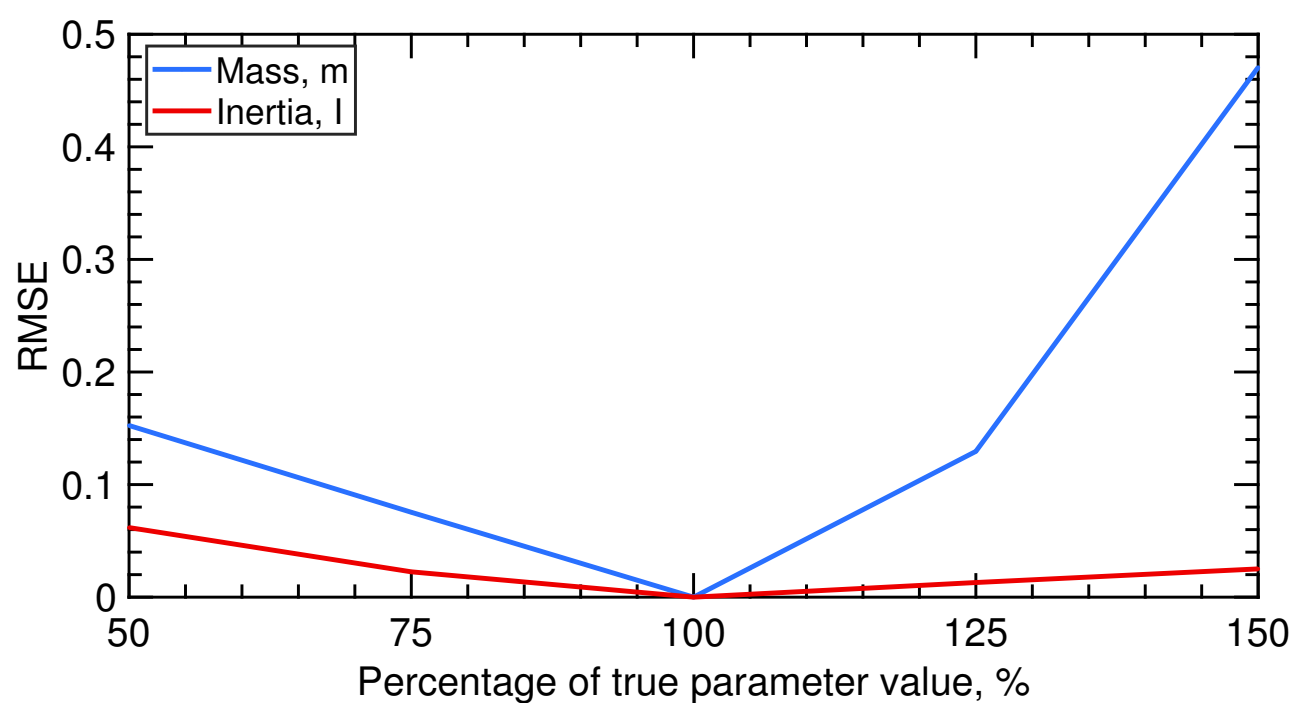

Figure 17. Root mean square error (RMSE) of the vehicle trajectory.

\subsubsection{Incorrect Resistance Coefficients}

In the next considered scenario, it is assumed that the longitudinal and lateral resistance coefficients vary depending on the $\mathrm{X}-\mathrm{Y}$ plane location. Such scenarios are relevant for the cases when vehicle is moving through unknown rugged terrain where different types of vehicle-terrain interactions can be met.

Maps of the coefficient distributions over the surface are synthesized based on the experimental data provided in Reference [5] and shown in Figure 18.

The dependencies of the resistance coefficients for the longitudinal and lateral coefficients were generated with the following functions, respectively:

$$
\mu_{l}=0.7+0.02 X \cos (0.3 Y)
$$

and

$$
\mu_{t}=0.6+0.03 X \sin (0.2 X) e^{-0.01 Y} .
$$

The functions (44) and (45) were selected quite arbitrarily, just to test the ability of the controller to cope with uncertainties.

Behavior of the coefficients along the vehicle trajectory is given in Figure 19 with solid lines. Reference values adopted in the controller are marked with the dotted lines. 

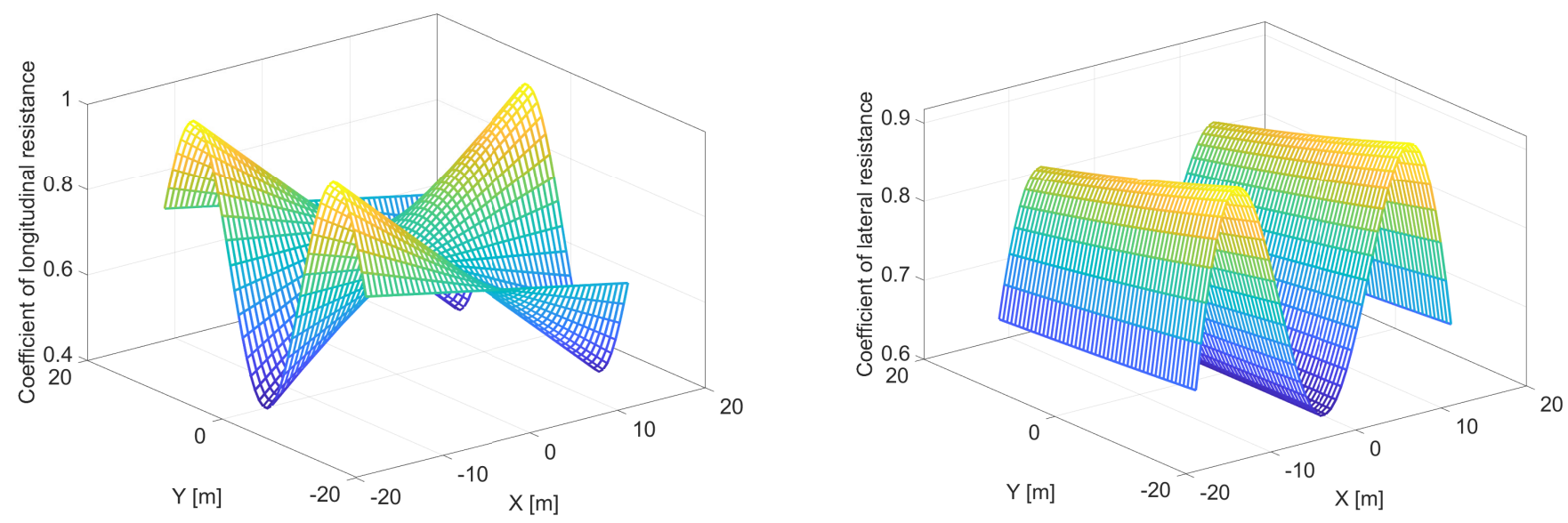

Figure 18. Maps of resistance coefficients: longitudinal (left) and lateral (right).

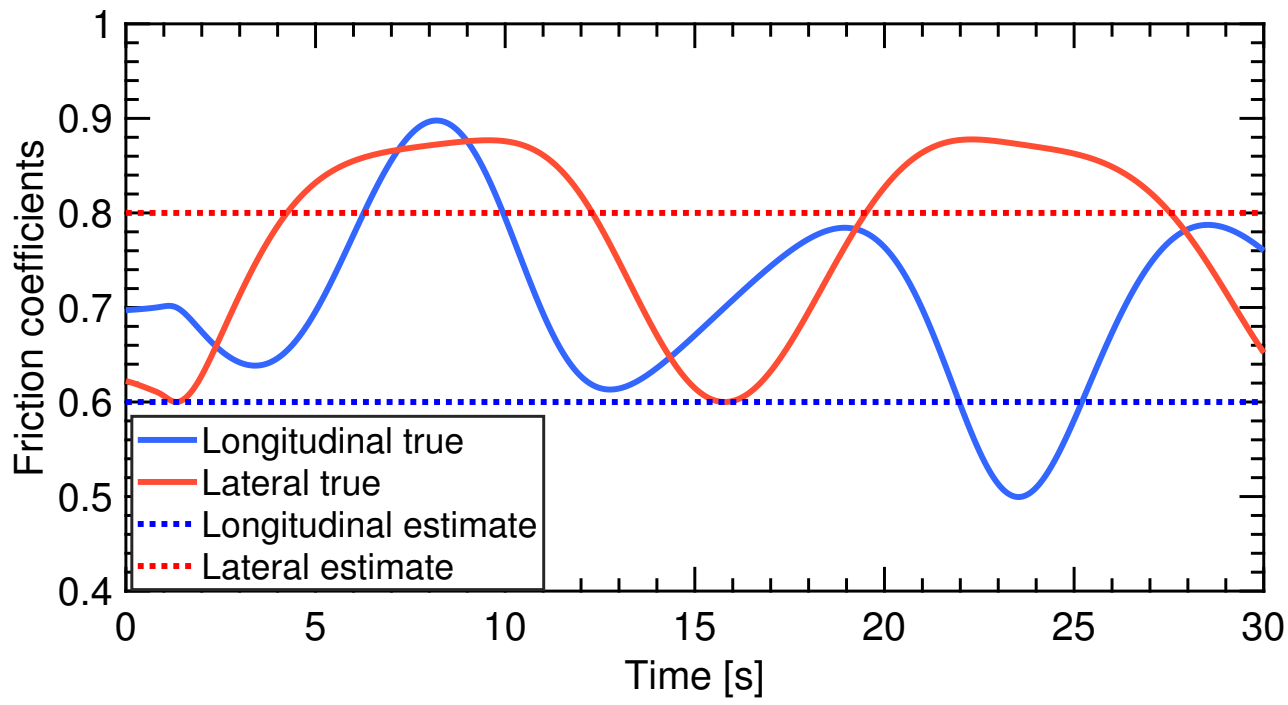

Figure 19. Friction coefficients values on the vehicle path (circular path).

The trajectory tracking tests under the discussed above variations of the resistance coefficients are provided in Figure 20.

From presented results, one can observe that the performance of the controller is more sensitive to the uncertainties in resistance coefficients for the rotational motion. Namely, in the circular test, vehicle trajectory diverges from the reference one for the sections of the path, where the real values of the coefficients differ significantly from the values assumed in the controller. The controller performance for the straight-line motion is still good enough even for the inaccurate coefficient approximations since the lateral resistance coefficient does not affect significantly on the trajectory tracking precision in the longitudinal direction and the longitudinal resistance coefficient uncertainty cause inaccuracies rather for the velocity.

The circular test is selected for a further analysis since the uncertainty in resistance coefficients can produce more effect on the system dynamics in this case.

Instantaneous forward and rotational velocities are given in Figure 21, while the controller errors are provided in Figure 22.

From the figures, one can conclude that the tracking errors are observed where the assumed friction model has mismatches with the true values (Figure 19).

The system parameter states, which are the torque, the sprocket rotational velocity, and the slip of the track, are demonstrated in Figure 23 and compared with the parameters 
when the correct coefficients are available. It can be noted that the controller cannot reach a steady-state, and the oscillatory response of the system reflects the changes in the resistant properties of the terrain.
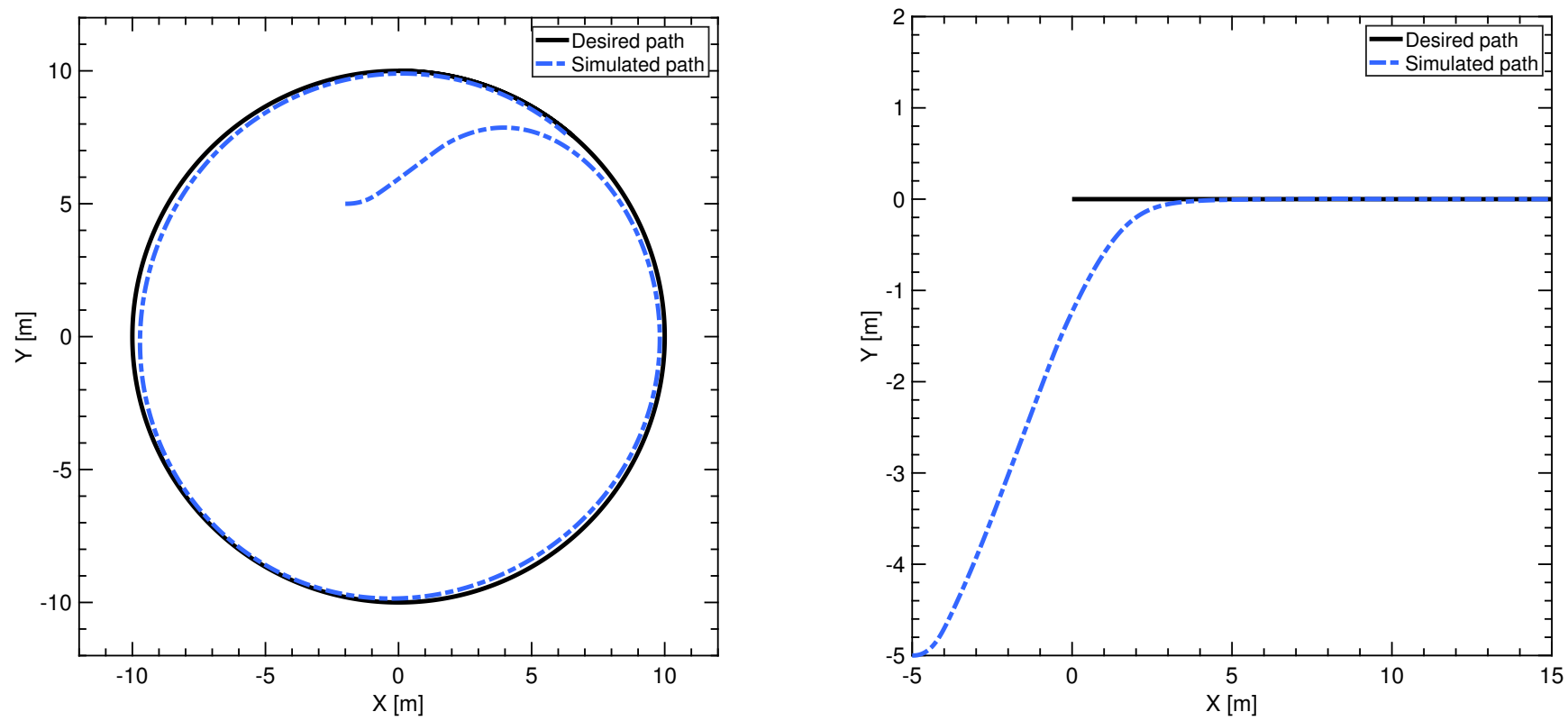

Figure 20. Trajectory following test in case of incorrect knowledge of resistance coefficients: circular (left) and straight paths (right).
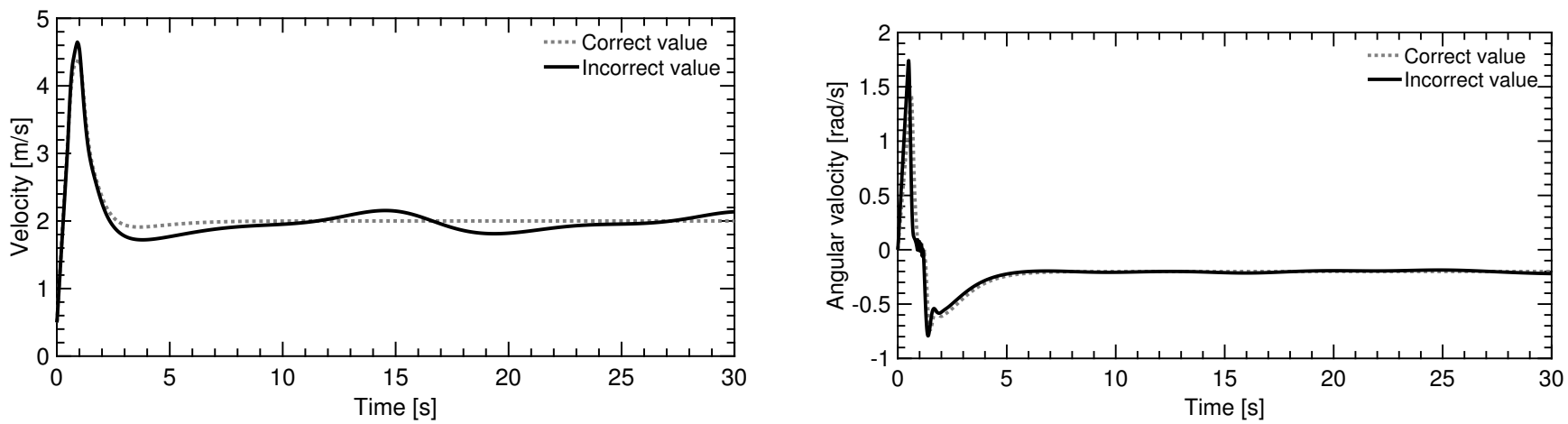

Figure 21. Trajectory following test in case of incorrect knowledge of resistance coefficients: instantaneous forward velocity (left) and rotational velocity (right).
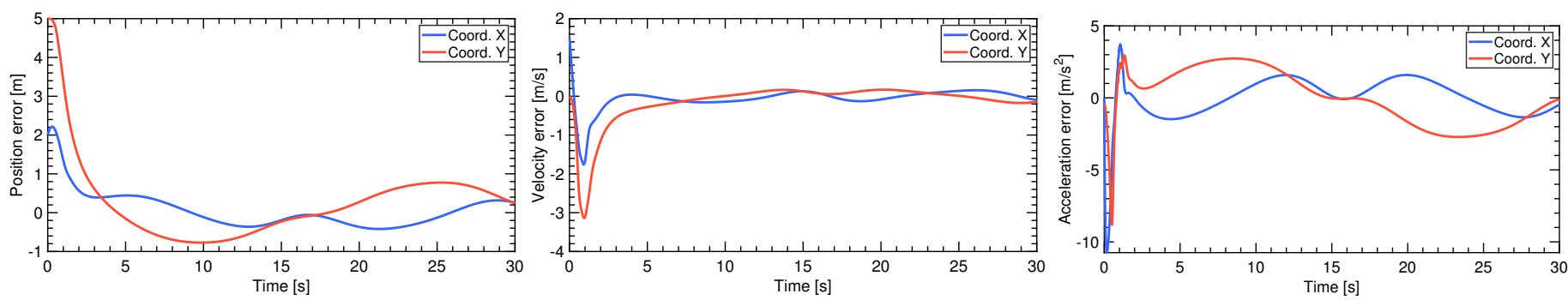

Figure 22. Trajectory following test (circular path) in case of incorrect knowledge of resistance coefficients: position error (left), velocity error (middle), and acceleration error (right). 

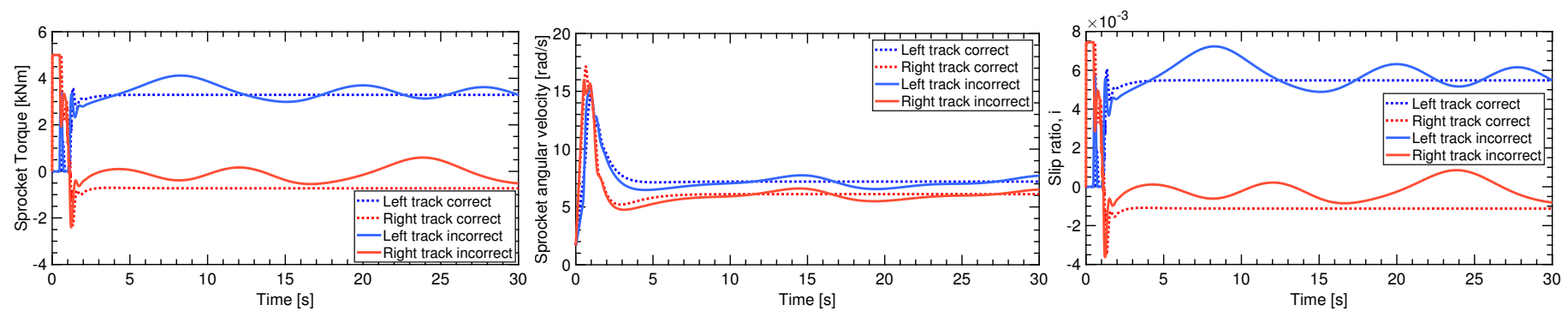

Figure 23. Trajectory following test (circular path) in case of incorrect knowledge of resistance coefficients: torque behavior (left), sprocket rotational velocity (middle), and slip of the track (right).

\subsubsection{Incorrect Estimation of Maximum Tractive Effort}

In this section, the last scenario is considered, namely the maximum tractive effort that the vehicle can develop on the certain type of soil is incorrectly predicted, which results in the trajectory demand that the vehicle is unable to follow. The expected maximum tractive effort was computed for the heavy clay (parameters provided in Table 2), while the actual parameters in this simulation corresponded to sandy loam with $c=9.65 \mathrm{kPa}$ and $\phi=35^{\circ}$.

In this scenario, we performed the same simulation tests, namely the circular and straight path following, which are shown in Figures 24 and 25.
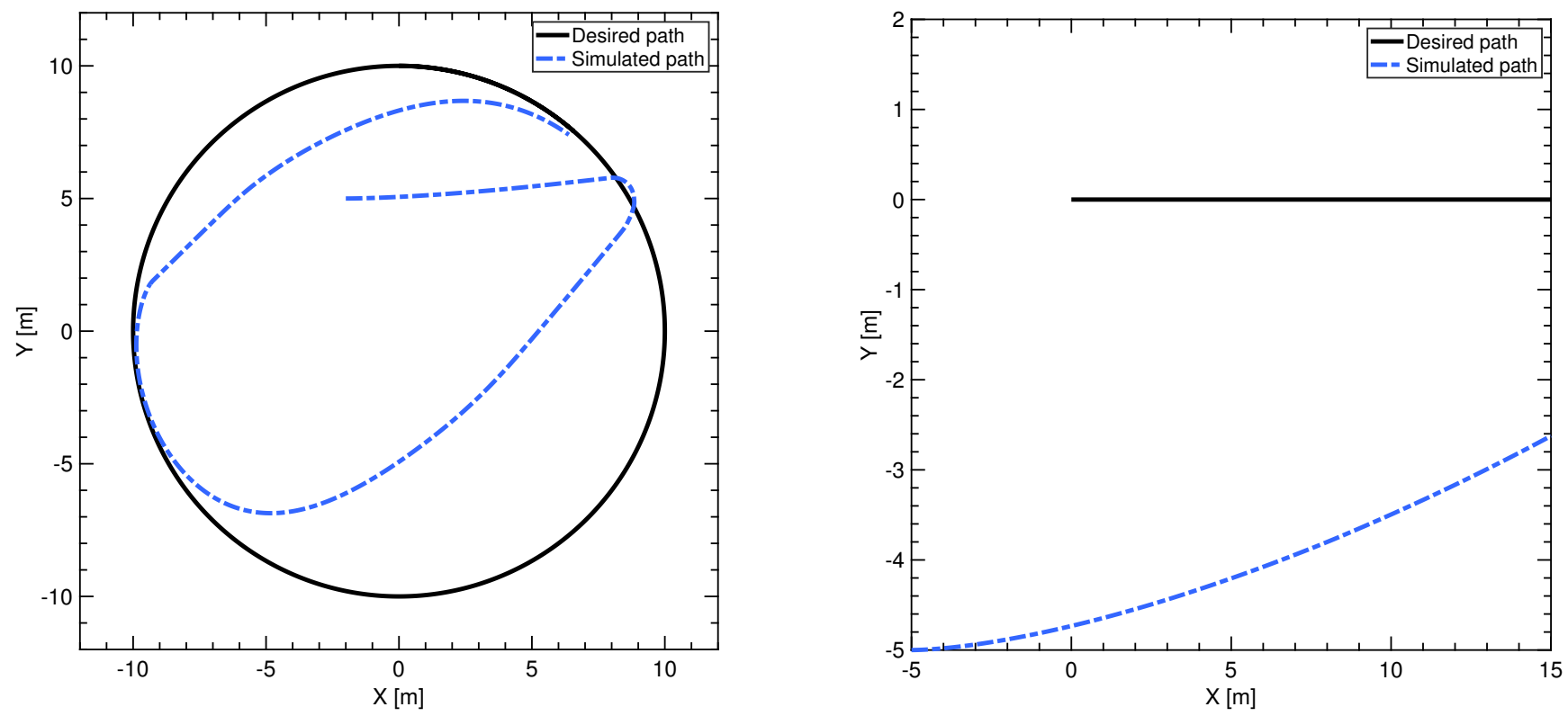

Figure 24. Trajectory following test in case of incorrect tractive force estimation: circular path (left) and straight path (right).
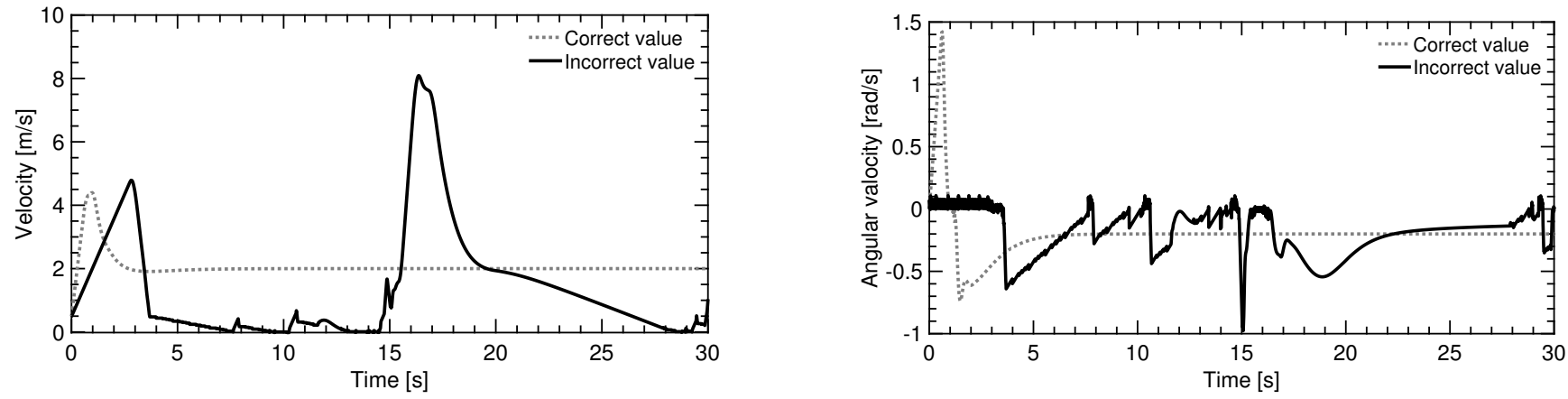

Figure 25. Trajectory following test in case of incorrect tractive force estimation: velocity $V$ (left) and rotational velocity $\omega$ (right). 
As it is clearly seen in the figures, the vehicle cannot follow the desired trajectory and causes large errors in positioning and following the desired velocities (Figure 26).
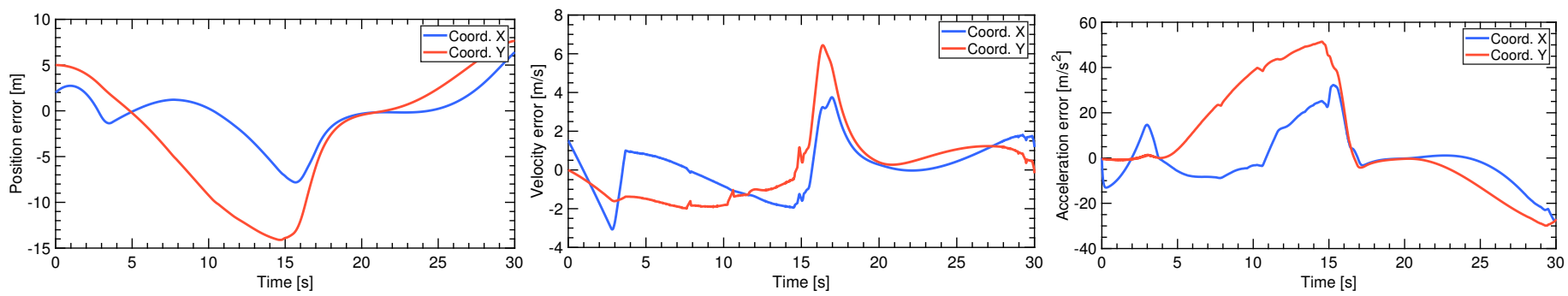

Figure 26. Trajectory following test (circular path) in case of incorrect tractive force estimation: position error (left), velocity error (middle), and acceleration error (right).

The demanded torque is too big for the system capabilities (Figure 27) and causes control saturation. The vehicle cannot provide proper acceleration to follow the circular path. The controller even is not capable of maintaining a steady velocity. From Figure 25, one can conclude that, in the 7th second of the simulation, the vehicle practically stops when it reaches the desired trajectory for the first time. Then, it rapidly accelerates after a couple of seconds to mitigate a huge position error, shown in Figure 26, that has been accumulated in the process.
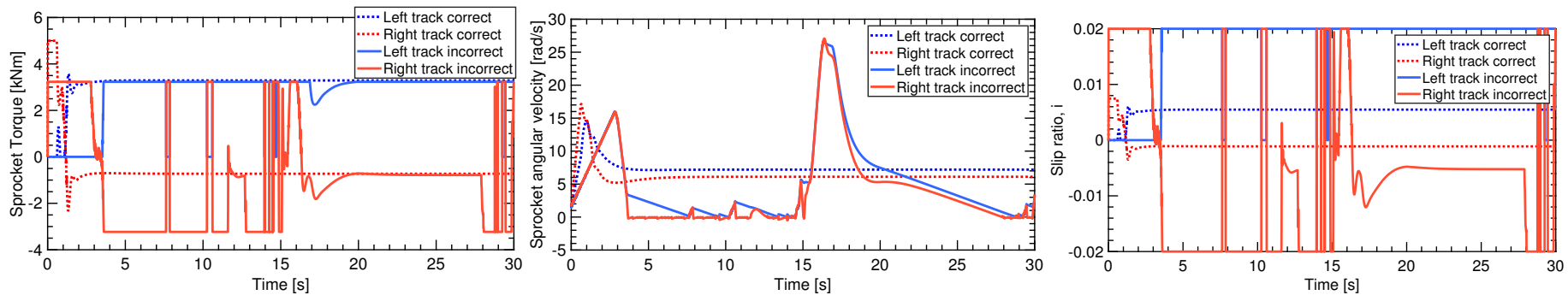

Figure 27. Trajectory following test (circular path) in case of incorrect tractive force estimation: torque behavior (left), sprocket rotational velocity (middle), and slip of the track (right).

To summarize this section, it can be concluded that incorrect estimation of the vehicle or soil-track interaction parameters can reduce the control system performance. As a result, the vehicle cannot follow the desired trajectory. In particular, inaccurate predictions of mass, inertia or friction coefficients introduce errors between the obtained trajectory of the vehicle and the reference trajectory. This, in turn, can cause extensive energy consumption as the motors have to generate bigger moments to guide the vehicle back on the path. Furthermore, a wrong assumption of the soil parameters can even lead to total inability to follow the desired trajectory.

To address the issues discussed above, the identification framework is implemented and tested. The results are presented in the next section.

\subsection{Validation of Parameters Identification}

This section presents results obtained with parameter identification algorithms, which were previously introduced in Section 7.

It should be indicated here that some of the identified parameters, such as mass, moment of inertia, and longitudinal and lateral resistance coefficients, can be used to adjust controller on the fly, thus providing an adaptive augmentation to the baseline controller. However, some of the parameters, which are track slip ratios and soil parameters can be used only for the trajectory modifications due to new environmental conditions, thus giving a contribution to an adaptive path planning framework. However, in the current study, we consider the trajectory to be predefined and unchangeable. So, the identified mass, moment of inertia, and longitudinal and lateral resistance coefficients are used to 
improve controller performance, and track slip ratios, maximum tractive force, and shear deformation are only identified and are not used to improve the motion performance of the vehicle.

\subsubsection{Identification of Mass, Moment of Inertia and Resistance Coefficients}

As discussed in Section 7.1.1, EF RLS is selected for identification of mass and moment of inertia of the vehicle. Forgetting factor $\lambda=0.998$ is utilized in the current simulations.

Identification of mass and moment of inertia is demonstrated in Figures 28 and 29. Identification of the longitudinal and lateral resistance coefficients is provided in Figures 30 and 31. The identification maneuver can be found in Figure 32.
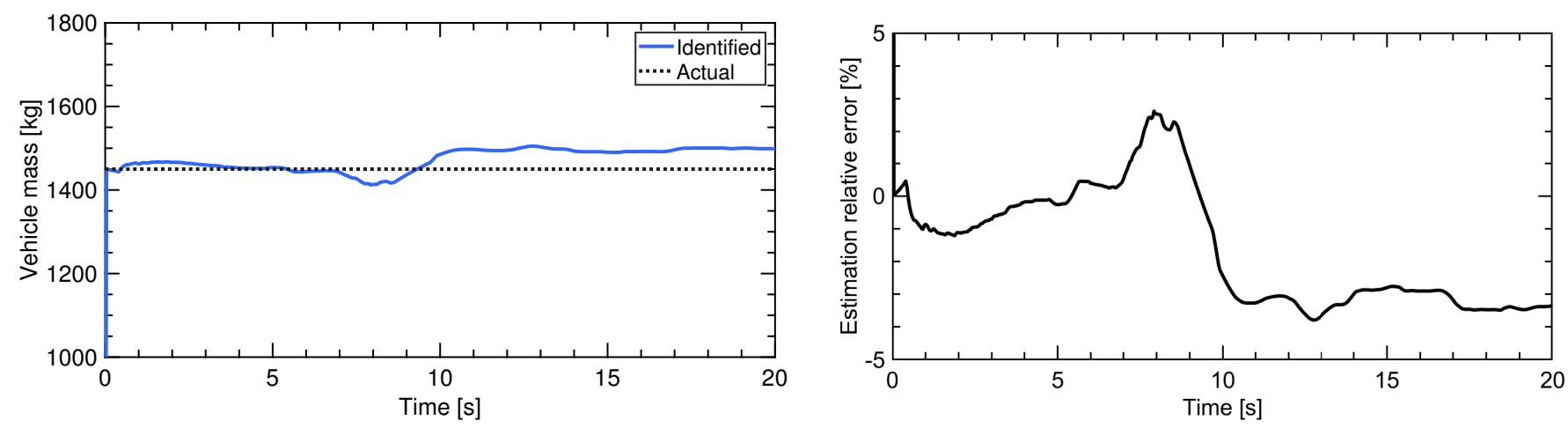

Figure 28. Identification of mass: identified value (left) and relative error (right).
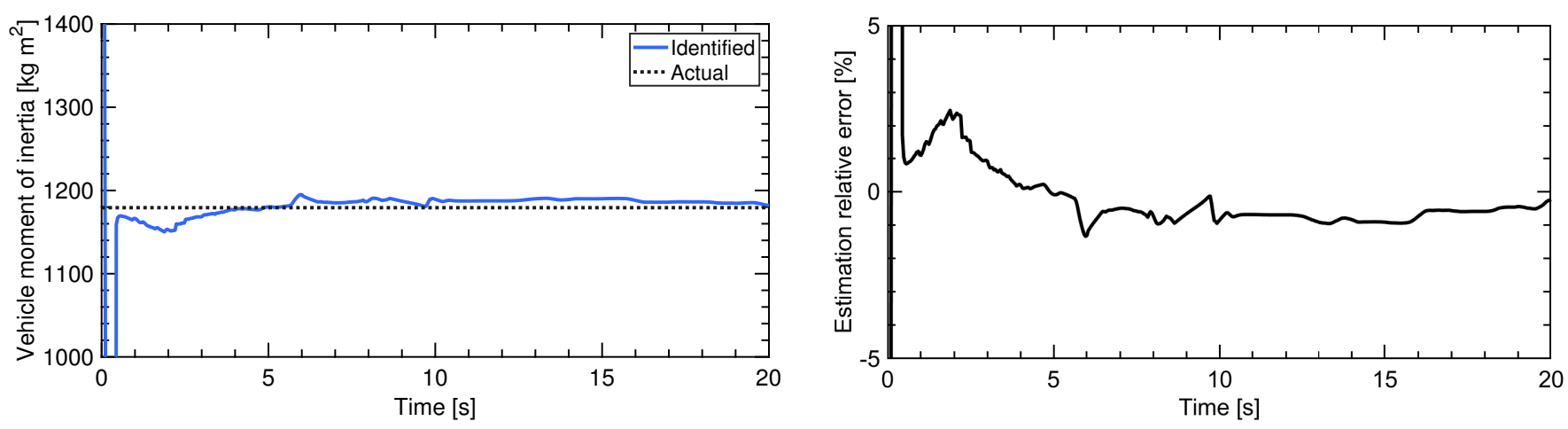

Figure 29. Identification of moment of inertia: identified value (left) and relative error (right).

The identified values were used to improve the controller performance under uncertainties provided earlier in Section 8.2. Data obtained with the identification scheme was then fed into the controller to form the adaptation capabilities of the control scheme. The trajectory tracking of the controller with adaptive augmentation is compared with the baseline controller in Figure 32. One can notice that the identification of the parameters decreases position error as compared to the baseline controller.

It can be observed that both mass and inertia of the vehicle were estimated quite accurately. However, predictions of moment of inertia is more accurate.

The precision of mass identification is better during the first $7 \mathrm{~s}$ (the error is less than $1 \%$ ), while the vehicle being driven along the straight path. The identification error increases after the vehicle starts the spiral motion. This is caused by the fact that the mass identification scheme developed in Section 7 assumes the longitudinal motion. Even under this assumption, the proposed scheme helps to to improve the trajectory tracking (Figure 32); in particular, the error is kept at the satisfactory level, namely below $5 \%$. The identification precision might be further improved either by changing the assumptions of longitudinal motion or by synchronization of the updating system parameters with 
the identification maneuvers by using some top level governing algorithm similar to that proposed in Reference [49].
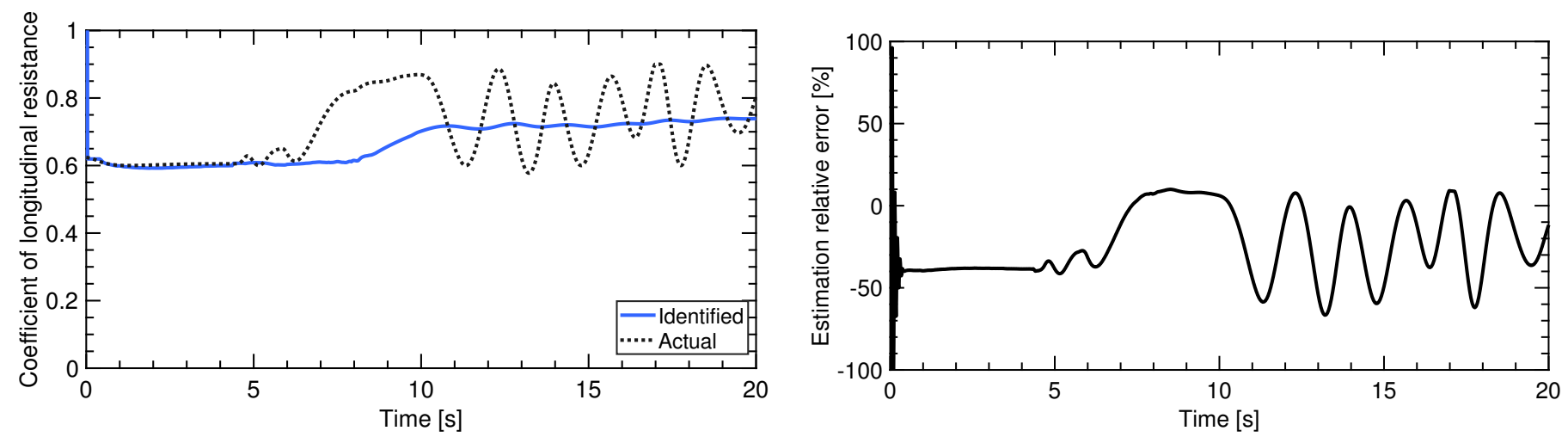

Figure 30. Longitudinal resistance coefficient: identified value (left) and relative error (right).
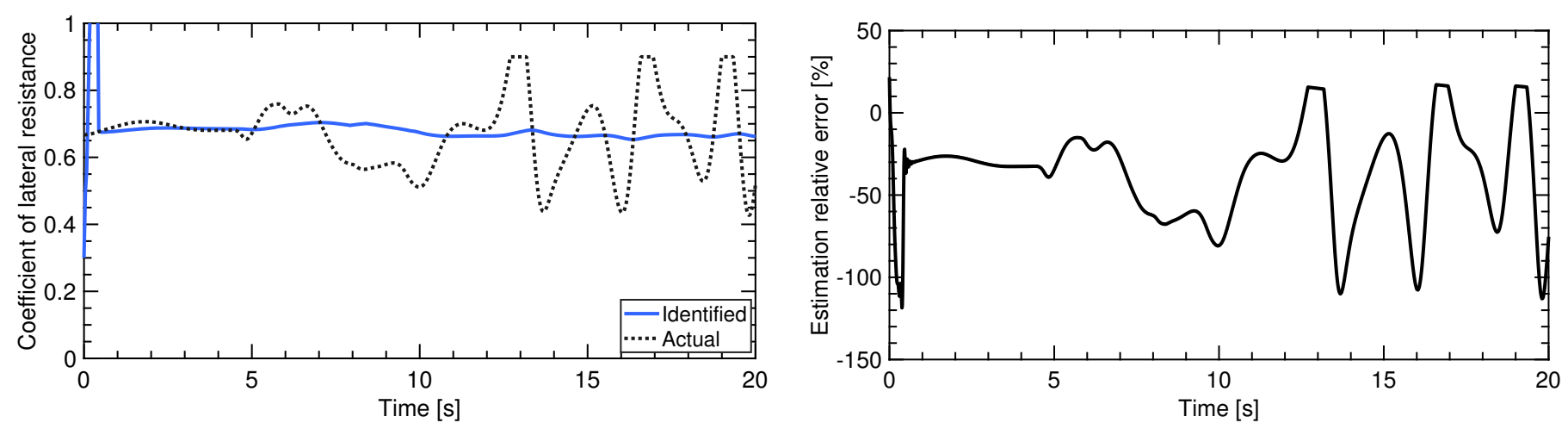

Figure 31. Lateral resistance coefficient: identified value (left) and relative error (right).

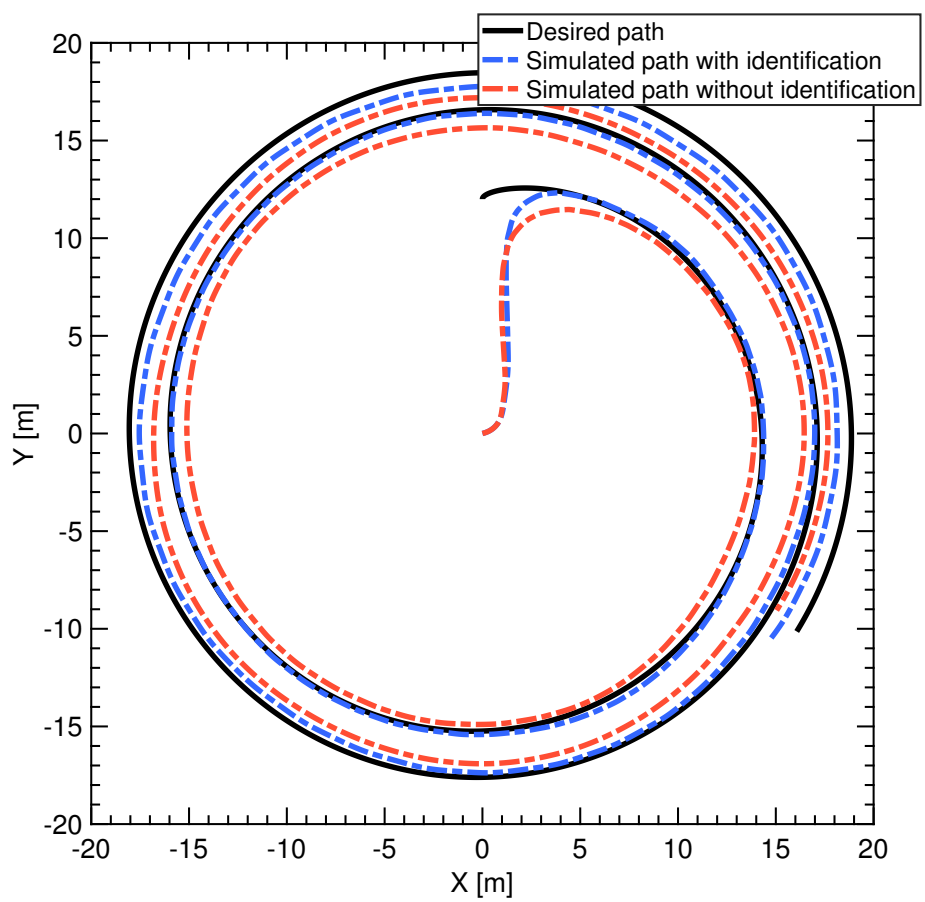

Figure 32. Trajectory of the vehicle: with identified parameters and incorrect parameters. 
Estimations of the coefficients of longitudinal and lateral resistance are at a quite reasonable level. The proposed scheme identifies the resistance coefficients quite good when the coefficients vary slowly. Starting from the $t=6 \mathrm{~s}$, the resistance coefficients start to change very fast and the EF RLS cannot follow such an abrupt variations. This is quite well known limitation of the RLS method. Nevertheless, the EF RLS provides good time average estimates of $\mu_{L}$ and $\mu_{t}$. The variation of the resistant coefficients, simulated in the current experiment (see Equations (44) and (45)), is not quite realistic, namely, in the real motion, the the resistance coefficients are not expected to vary so fast; thus, the proposed scheme should be applicable for real-time applications.

\subsubsection{Identification of Slip Parameter}

Estimation of the slip parameter is performed with the UKF-based approach, which utilizes kinematics of the vehicle for state equations and is described in Section 7.2. Identification of the track slip ratio together with the actual value is coplotted in Figure 33.
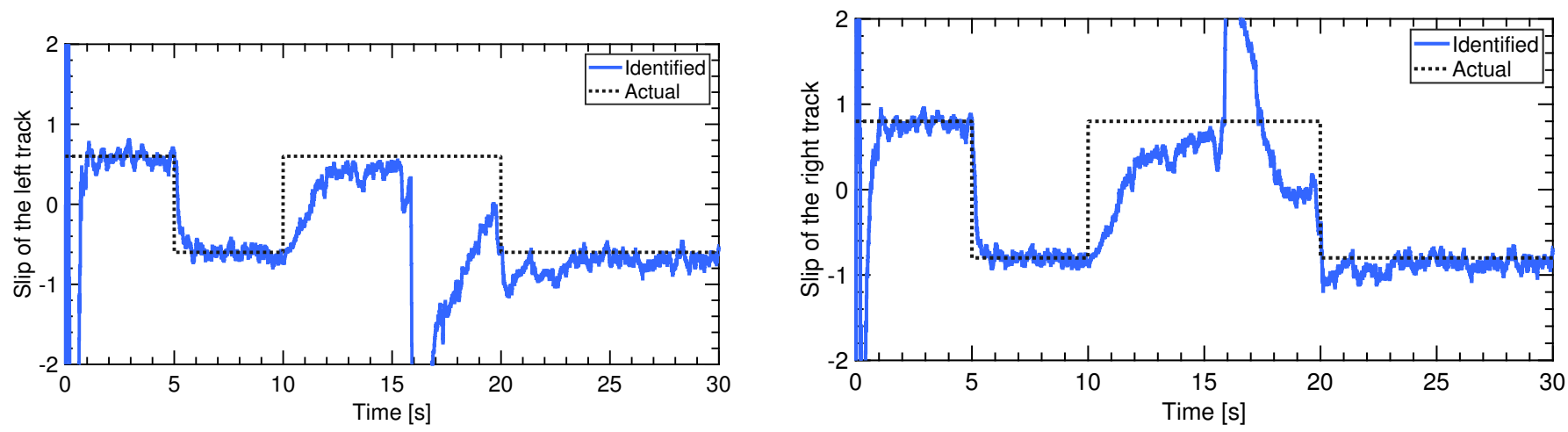

Figure 33. Track slip ratio identification: Left track (left) and Right track (right).

In the simulation, UKF and controller are fed with noisy measurement data. It can be noticed that the filter is capable of capturing the changing values of the slip ratio for both the left and right track. It provides an unbiased estimation of the states. The temporary divergence from the actual value between 15th and 20th second of the simulation was caused by the rapid speed change of the vehicle. Thus, for a relatively slow motion of the vehicle, the proposed approach manifests quite a good result.

\subsubsection{Identification of Soil Parameters}

Soil parameters, i.e., maximum tractive force and shear deformation parameter were identified using the GNR method presented in Section 7.3. Results are shown in Figures 34 and 35 . It can be concluded that the algorithm is able to obtain very accurate estimations of the parameters and adapts to the changes almost instantly.
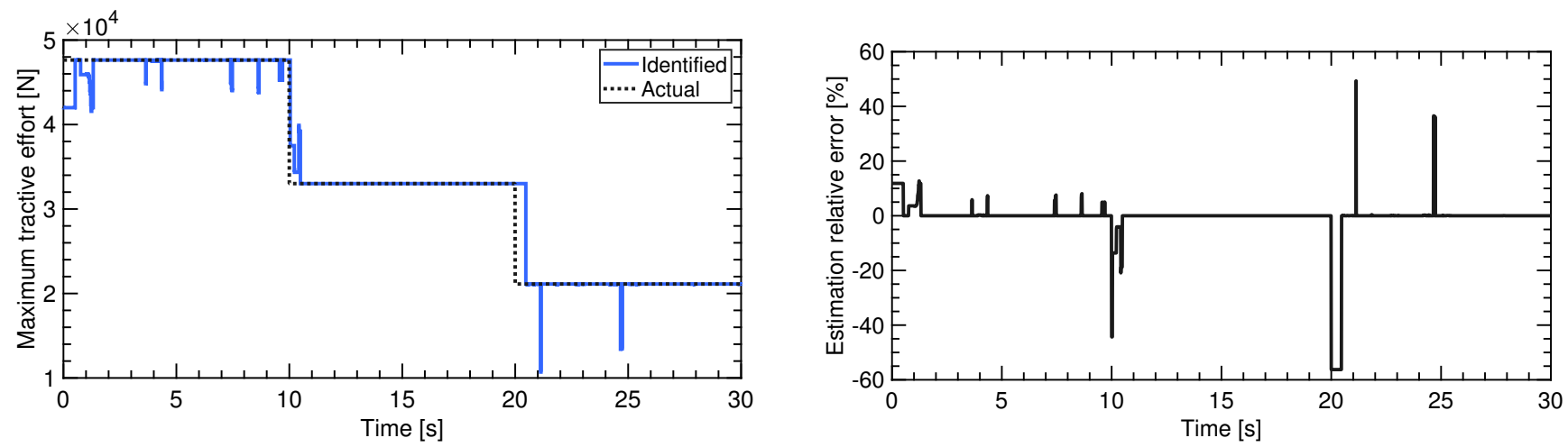

Figure 34. Identification of max. tractive force: identified value (left) and relative error (right). 

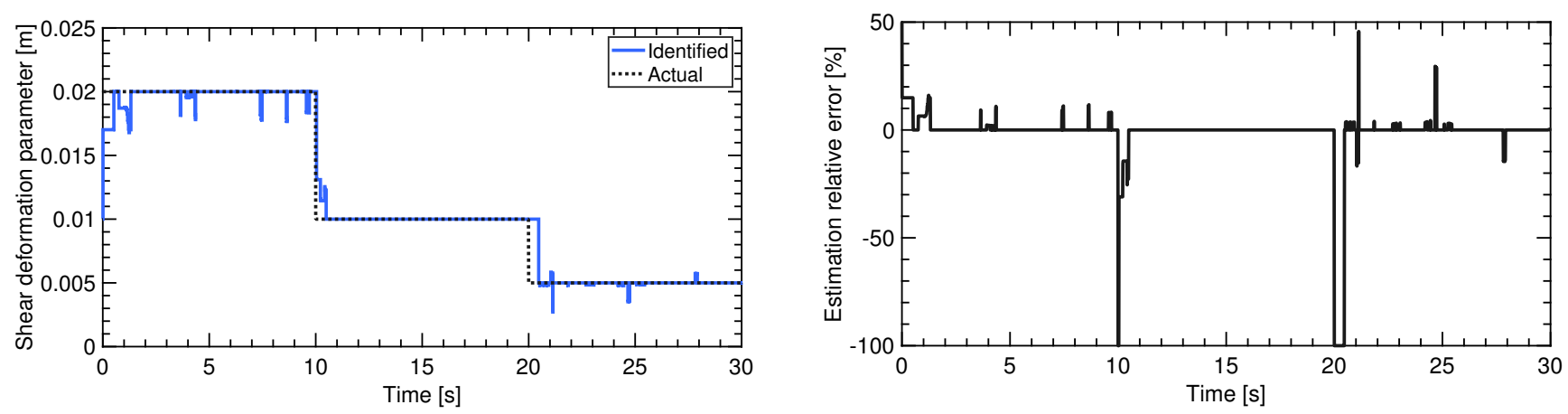

Figure 35. Identification of shear deformation parameter: identified value (left) and relative error (right).

Different settings of the window size for the number of measurement samples were tested. It was concluded that fifty samples provided robust behavior and were not computationally demanding at the same time. Additionally, the maximum number of iterations was set to twenty. In case when the algorithm was unable to converge, the last converged value was provided at the output. The number of iterations for each time step is presented in Figure 36.

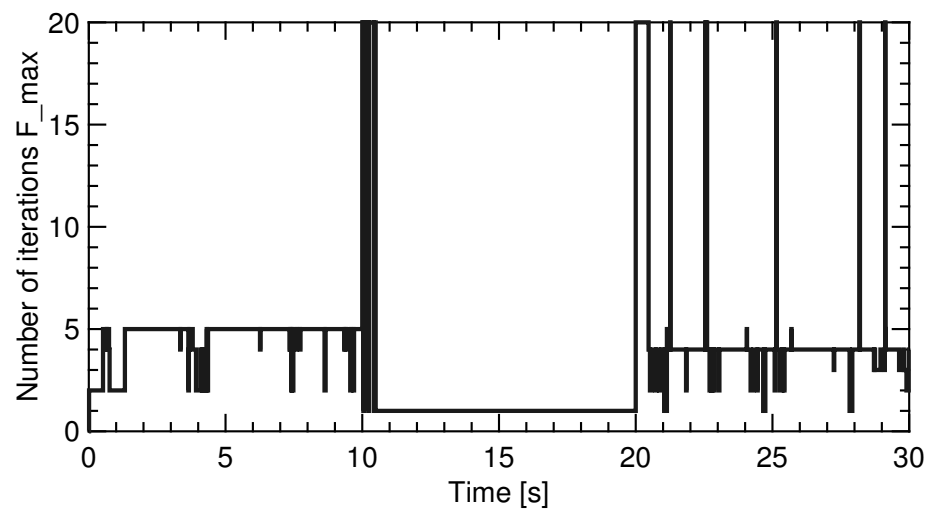

Figure 36. Generalized Newton-Raphson (GNR)—number of algorithm iterations.

Comparing the prediction error (Figures 34 and 35) with the number of iterations in Figure 36 one can conclude that high error is mainly obtained when the algorithm was unable to converge. Thus, fine-tuning of the number of iterations could further improve the algorithm performance. However, it can be done only by sacrificing the computational power of the on-board computer and should be decided for a particular real-world application based on its characteristics.

It should be noted that conversely to the inertial parameters, soil parameters identification yields better results for the experiment conducted when the vehicle is moving in a steady-state and not performing rapid maneuvers. This illustrates once again the need for different identification methods (including different maneuvers) for different state parameters and the presence of a governing algorithm to supervise learning [49].

\section{Conclusions}

The increasing level of autonomy of unmanned ground vehicle dictates a higher demand for operation performance, including trajectory tracking precision. Presence of model uncertainties can significantly reduce ground vehicle performance when then vehicle is traversing an unknown terrain or the vehicle inertial parameters are changed due to a mission schedule or external disturbances. Current research addressed the problem of trajectory tracking capabilities of a tracked vehicle under uncertainties in inertial parameters and in the vehicle-terrain interaction model. 
At the beginning, a deep insight into the dynamics of a tracked vehicle was provided, which facilitates better understanding of the system vulnerabilities and possible control problems.

Next, the controller aiming to obtain trajectory tracking capabilities was developed. The control system design tackled the challenges brought by the nonlinear nature of the tracked vehicle dynamics. In case of perfect knowledge of motion parameters, the proposed control law perfectly follows the desired trajectory. However, when the vehicle model parameters are unknown or incorrectly estimated, the vehicle closed-loop dynamics will still be nonlinear. The influence of an uncertainties in the system dynamics is a torque disturbance that should be compensated by a proper control design.

Sensitivity of the controller to the model uncertainties was analysed and provided the valuable intuition into the consequences, such as controller divergence, offset from the desired trajectory, etc. It was shown that a precise approximation of the parameters employed in the control scheme improved tracking capabilities.

In addition, three identification schemes were proposed to address different issues of parameters estimation. First, the EF RLS was utilized to obtain improved estimates of the mass and moment of inertia of the vehicle, as well as friction coefficients. The algorithm yields very good estimation of the vehicle mass and inertia. Regarding the friction coefficients, the RLS method adapted at a slower pace to the dynamically changing terrain characteristics still providing an acceptable average from the process.

Identified values were further used to form an adaptive augmentation loop and to improve the tracking performance of the controller. It was demonstrated that providing estimations to the controller helps to follow the desired path more precisely.

Next, the Unscented Kalman Filter was designed to estimate the slip ratio of the tracks. Only kinematics of the vehicle were included in the state equation. The filter was able to track the actual slip values accurately.

Finally, the generalized Newton-Raphson algorithm was employed for accurate estimations of the soil parameters.

The estimated values of vehicle-terrain interaction did not effect directly on the controller; thus, knowledge of these parameters was not used to improve the control performance. Such parameters can be used for improved path and trajectory planning in the future; however, for the purposes of this work, the trajectory was assumed to be defined a priori.

The proposed approach helps to improve vehicle tracking capabilities regardless of a control approach via identifying uncertainties of the tracked vehicle dynamics. The developed framework can tackle the trajectory tracking problem not only as a direct problem but also as a reciprocal problem. Indeed, the identification module provides estimations of uncertain parameters to the controller for appropriate trajectory tracking. At the same time, estimated vehicle-terrain interaction parameters can be used for generating feasible trajectories. Such bilateral tackling of trajectory tracking is essential for future autonomous vehicle missions.

Future work will look into the algorithms proposed in the paper towards development of an adaptive trajectory planner and validation on a testbed.

Author Contributions: The authors contributed to the article as follows: conceptualization, N.S., D.I.I. and A.T.; methodology, N.S. and D.I.I.; software, N.S.; validation, N.S.; investigation, N.S.; writing—original draft preparation, N.S. and D.I.I.; writing—review and editing, N.S., D.I.I., A.C.Z. and A.T.; supervision, D.I.I. and A.T.; project administration, A.T. All authors have read and agreed to the published version of the manuscript.

Funding: This research received no external funding.

Acknowledgments: The authors would like to thank Jeremy Baxter from QinetiQ for fruitful discussions helped to crystallize the problem statement and for providing thoughtful comments on the research.

Conflicts of Interest: The authors declare no conflict of interest. 


\section{References}

1. Astrom, K.J.; Wittenmark, B. Adaptive Control, 2nd ed.; Addison-Wesley Longman Publishing Co., Inc.: Petaluma, CA, USA, 1994.

2. Wellhausen, L.; Dosovitskiy, A.; Ranftl, R.; Walas, K.; Cadena, C.; Hutter, M. Where Should I Walk? Predicting Terrain Properties From Images Via Self-Supervised Learning. IEEE Robot. Autom. Lett. 2019, 4, 1509-1516. [CrossRef]

3. Shiller, Z.; Serate, W.; Hua, M. Trajectory planning of tracked vehicles. In Proceedings of the IEEE International Conference on Robotics and Automation, Atlanta, GA, USA, 2-6 May 1993; Volume 3, pp. 796-801.

4. Ma, Y.; Li, Y.; Liang, H. Design of sliding mode controller on steering control of skid steering $6 \times 6$ unmanned vehicle. In Proceedings of the 2017 IEEE International Conference on Unmanned Systems (ICUS), Beijing, China, 27-29 October 2017; pp. 272-276.

5. Wong, J.Y. Theory of Ground Vehicles, 4th ed.; Wiley: Hoboken, NJ, USA, 2008.

6. Elshazly, O.; Abo-Ismail, A.; Abbas, H.S.; Zyada, Z. Skid steering mobile robot modeling and control. In Proceedings of the 2014 UKACC International Conference on Control (CONTROL), Loughborough, UK, 9-11 July 2014; pp. 62-67.

7. Wu, Y.; Wang, T.; Liang, J.; Chen, J.; Zhao, Q.; Yang, X.; Han, C. Experimental kinematics modeling estimation for wheeled skid-steering mobile robots. In Proceedings of the 2013 IEEE International Conference on Robotics and Biomimetics (ROBIO), Shenzhen, China, 12-14 December 2013; pp. 268-273.

8. Sutoh, M.; Iijima, Y.; Sakakieda, Y.; Wakabayashi, S. Motion Modeling and Localization of Skid-Steering Wheeled Rover on Loose Terrain. IEEE Robot. Autom. Lett. 2018, 3, 4031-4037. [CrossRef]

9. Pacejka, H.B. Tire and Vehicle Dynamics; Butterworth-Heinemann Distributed in conjunction with SAE International: Oxford, UK; Waltham, MA, USA; Warrendale, PA, USA, 2012.

10. Meng, H.; Xiong, L.; Gao, L.; Yu, Z.; Zhang, R. Tire-Model-Free Control for Steering of Skid Steering Vehicle. In Proceedings of the 2018 IEEE Intelligent Vehicles Symposium (IV), Changshu, China, 26-30 June 2018; pp. 1590-1595.

11. Caracciolo, L.; De Luca, A.; Iannitti, S. Trajectory tracking control of a four-wheel differentially driven mobile robot. In Proceedings of the 1999 IEEE International Conference on Robotics and Automation (Cat. No.99CH36288C), Detroit, MI, USA, 10-15 May 1999; Volume 4, pp. 2632-2638.

12. Kozlowski, K.; Pazderski, D. Modeling and control of a 4-wheel skid-steering mobile robot. Int. J. Appl. Math. Comput. Sci. 2004, 14, 477-496.

13. Zou, T.; Angeles, J.; Hassani, F. Dynamic modeling and trajectory tracking control of unmanned tracked vehicles. Robot. Auton. Syst. 2018, 110, 102-111. [CrossRef]

14. Ahmadi, M.; Polotski, V.; Hurteau, R. Path tracking control of tracked vehicles. In Proceedings of the 2000 ICRA. Millennium Conference. IEEE International Conference on Robotics and Automation. Symposia Proceedings (Cat. No.00CH37065), San Francisco, CA, USA, 24-28 April 2000; Volume 3, pp. 2938-2943.

15. Tang, S.; Yuan, S.; Hu, J.; Li, X.; Zhou, J.; Guo, J. Modeling of steady-state performance of skid-steering for high-speed tracked vehicles. J. Terramech. 2017, 73, 25-35. [CrossRef]

16. Economou, J.T.; Colyer, R.E. Modelling of skid steering and fuzzy logic vehicle ground interaction. In Proceedings of the 2000 American Control Conference, ACC (IEEE Cat. No.00CH36334), Chicago, IL, USA, 28-30 June 2000; Volume 1, pp. $100-104$.

17. Garimella, G.; Funke, J.; Wang, C.; Kobilarov, M. Neural network modeling for steering control of an autonomous vehicle. In Proceedings of the 2017 IEEE/RSJ International Conference on Intelligent Robots and Systems (IROS), Vancouver, BC, Canada, 24-28 September 2017; pp. 2609-2615.

18. De Luca, A.; Oriolo, G.; Samson, C. Feedback control of a nonholonomic car-like robot. In Robot Motion Planning and Control; Laumond, J.P., Ed.; Springer: Berlin/Heidelberg, Germany, 1998; pp. 171-253.

19. Inoue, R.S.; Cerri, J.P.; Terra, M.H.; Siqueira, A.A.G. Robust recursive control of a skid-steering mobile robot. In Proceedings of the 2013 16th International Conference on Advanced Robotics (ICAR), Montevideo, Uruguay, 25-29 November 2013; pp. 1-6.

20. Liu, J.; Han, W.; Liu, C.; Peng, H. A New Method for the Optimal Control Problem of Path Planning for Unmanned Ground Systems. IEEE Access 2018, 6, 33251-33260. [CrossRef]

21. Liu, J.; Han, W.; Zhang, Y.; Chen, Z.; Peng, H. Design of an Online Nonlinear Optimal Tracking Control Method for Unmanned Ground Systems. IEEE Access 2018, 6, 65429-65438. [CrossRef]

22. Jun, J.; Hua, M.; Benamar, F. A trajectory tracking control design for a skid-steering mobile robot by adapting its desired instantaneous center of rotation. In Proceedings of the 53rd IEEE Conference on Decision and Control, Los Angeles, CA, USA, 15-17 December 2014; pp. 4554-4559.

23. Lin, N.; Zong, C.; Shi, S. The Method of Mass Estimation Considering System Error in Vehicle Longitudinal Dynamics. Energies 2018, 12, 52. [CrossRef]

24. Vahidi, A.; Stefanopoulou, A.; Peng, H. Recursive least squares with forgetting for online estimation of vehicle mass and road grade: Theory and experiments. Veh. Syst. Dyn. 2005, 43, 31-55. [CrossRef]

25. Hsu, L.Y.; Chen, T.L. Vehicle Dynamic Prediction Systems with On-Line Identification of Vehicle Parameters and Road Conditions. Sensors 2012, 12, 15778-15800. [CrossRef] [PubMed]

26. Rhode, S.; Gauterin, F. Vehicle mass estimation using a total least-squares approach. In Proceedings of the 201215 th International IEEE Conference on Intelligent Transportation Systems, Anchorage, AK, USA, 16-19 September 2012; pp. $1584-1589$.

27. Dar, T.M.; Longoria, R.G. Slip estimation for small-scale robotic tracked vehicles. In Proceedings of the 2010 American Control Conference, Baltimore, MD, USA, 30 June-2 July 2010; pp. 6816-6821. 
28. Zhou, B.; Peng, Y.; Han, J. UKF based estimation and tracking control of nonholonomic mobile robots with slipping. In Proceedings of the 2007 IEEE International Conference on Robotics and Biomimetics (ROBIO), Sanya, China, 15-18 December 2007; pp. 2058-2063.

29. Cui, M.; Liu, W.; Liu, H.; Lü, X. Unscented Kalman Filter-based adaptive tracking control for wheeled mobile robots in the presence of wheel slipping. In Proceedings of the 2016 12th World Congress on Intelligent Control and Automation (WCICA), Guilin, China, 12-15 June 2016; pp. 3335-3340.

30. Song, Z.; Zweiri, Y.H.; Seneviratne, L.D.; Althoefer, K. Non-linear observer for slip estimation of tracked vehicles. Proc. Inst. Mech. Eng. Part D J. Automob. Eng. 2008, 222, 515-533. [CrossRef]

31. Song, Z.; Hutangkabodee, S.; Zweiri, Y.H.; Seneviratne, L.D.; Althoefer, K. Identification of soil parameters for unmanned ground vehicles track-terrain interaction dynamics. In Proceedings of the SICE 2004 Annual Conference, Sapporo, Japan, 4-6 August 2004; Volume 3, pp. 2255-2260.

32. Hutangkabodee, S.; Zweiri, Y.H.; Seneviratne, L.D.; Altho, K. Multi-solution Problem for Track-Terrain Interaction Dynamics and Lumped Soil Parameter Identification. In Field and Service Robotics; Corke, P., Sukkariah, S., Eds.; Springer: Berlin/Heidelberg, Germany, 2006; pp. 517-528.

33. Hutangkabodee, S.; Zweiri, Y.H.; Seneviratne, L.D.; Althoefer, K. Validation of Soil Parameter Identification for Track-Terrain Interaction Dynamics. In Proceedings of the 2007 IEEE/RSJ International Conference on Intelligent Robots and Systems, San Diego, CA, USA, 29 October-2 November 2007; pp. 3174-3179.

34. Angelova, A.; Matthies, L.; Helmick, D.; Perona, P. Learning and prediction of slip from visual information. J. Field Robot. 2007, 24, 205-231. [CrossRef]

35. Helmick, D.; Angelova, A.; Matthies, L. Terrain Adaptive Navigation for planetary rovers. J. Field Robot. 2009, 26, 391-410. [CrossRef]

36. Bekker, M. Introduction to Terrain-Vehicle Systems; University of Michigan Press: Ann Arbor, MI, USA, 1969.

37. Janosi, Z.; Hanamoto, B. Istituto elettrotecnico nazionale Galileo Ferraris. The Analytical Determination of Drawbar Pull as a Function of Slip for Tracked Vehicles in Deformable Soils. In Proceedings of the 1st International Conference of Terrain-Vehicle Systems, Turin, Italy, 1 June 1961; pp. 707-726.

38. Landau, L.; Lifshitz, E. Chapter VI-Motion of a Rigid Body. In Mechanics, 3rd ed.; Landau, L., Lifshitz, E., Eds.; ButterworthHeinemann: Oxford, UK, 1976; pp. 96-130. [CrossRef]

39. Choset, H.; Burgard, W.; Hutchinson, S.; Kantor, G.; Kavraki, L.E.; Lynch, K.M.; Thrun, S. Principles of Robot Motion: Theory, Algorithms, and Implementation; MIT Press: Cambridge, MA, USA, 2005.

40. Baruh, H. Analytical Dynamics; WCB/McGraw-Hill: Boston, MA, USA, 1999.

41. Ignatyev, D.I.; Shin, H.S.; Tsourdos, A. Bayesian calibration for multiple source regression model. Neurocomputing 2018, 318, 55-64. [CrossRef]

42. Grewal, M. Global Positioning Systems, Inertial Navigation, and Integration; Wiley-Interscience: Hoboken, NJ, USA, 2007.

43. Kionix. Using Two Tri-Axis Accelerometers for Rotational Measurements. 2015. Available online: http://kionixfs.kionix.com/en/ document/AN019\%20Using\%20Two\%20Tri-Axis\%20Accelerometers\%20for\%20Rotational\%20Measurements.pdf (accessed on 11 January 2021).

44. Soderstrom, T.; Stoica, P. System Identification (Prentice Hall International Series in Systems and Control Engineering); Prentice Hall: Englewood Cliffs, NJ, USA, 1989.

45. Van Der Merwe, R.; Doucet, A.; De Freitas, N.; Wan, E. The Unscented Particle Filter. In NIPS'00: Proceedings of the 13th International Conference on Neural Information Processing Systems; MIT Press: Cambridge, MA, USA, 2000; pp. 563-569.

46. Julier, S.J.; Uhlmann, J.K. New extension of the Kalman filter to nonlinear systems. In Proceedings of the Signal Processing, Sensor Fusion, and Target Recognition VI, Orlando, FL, USA, 21-24 April 1997; Volume 3068.

47. Zweiri, Y.H.; Seneviratne, L.D.; Althoefer, K. Parameter estimation for excavator arm using generalized Newton method. IEEE Trans. Robot. 2004, 20, 762-767. [CrossRef]

48. Zweiri, Y.H. Identification schemes for unmanned excavator arm parameters. Int. J. Autom. Comput. 2008, 5, 185-192. [CrossRef]

49. Ignatyev, D.I.; Shin, H.S.; Tsourdos, A. Two-layer adaptive augmentation for incremental backstepping flight control of transport aircraft in uncertain conditions. Aerosp. Sci. Technol. 2020, 105, 106051. [CrossRef] 